\title{
Half-hypermultiplets and incomplete/complete resolutions in F-theory
}

\author{
Naoto Kan, ${ }^{a}$ Shun'ya Mizoguchi ${ }^{a, b}$ and Taro Tani ${ }^{c}$ \\ ${ }^{a}$ Graduate University for Advanced Studies (Sokendai), \\ Tsukuba, Ibaraki, 305-0801, Japan \\ ${ }^{b}$ Theory Center, Institute of Particle and Nuclear Studies, KEK, \\ Tsukuba, Ibaraki, 305-0801, Japan \\ ${ }^{c}$ Kurume National College of Technology, \\ Kurume, Fukuoka, 830-8555, Japan \\ E-mail: naotok@post.kek.jp, mizoguch@post.kek.jp, \\ tani@kurume-nct.ac.jp
}

ABSTRACT: We consider resolutions of codimension-two enhanced singularities from $\mathrm{SO}(12)$ to $E_{7}$ and from $E_{7}$ to $E_{8}$ in six-dimensional F-theory, where a half-hypermultiplet arises for generic complex structures achieving them. The exceptional fibers at the enhanced point exhibit different structures depending on how the colliding 7-brane approaches the stack of gauge 7-branes, as previously observed by Morrison and Taylor in the case of the enhancement from $\mathrm{SU}(6)$ to $E_{6}$. When the colliding brane approaches them as $\mathrm{O}(s)$, where $s$ is the coordinate of the base space along the gauge 7-branes, the resolution process ends up with fewer exceptional fibers than naively expected from the Kodaira classification, with a non-Dynkin intersection matrix including half-integral intersection numbers. We confirm that the exceptional fibers at the enhanced point form extremal rays of the cone of the positive weights of the relevant pseudo-real representation, explaining why a halfhypermultiplet arises there. By altering the ordering of the singularities blown up in the process, we obtain, for both $\mathrm{SO}(12) \rightarrow E_{7}$ and $E_{7} \rightarrow E_{8}$, the intersection diagram on every other row of the corresponding box graphs. We present detailed derivations of the intersection diagrams of the exceptional fibers at the singularity enhanced points by examining how an exceptional curve is lifted up on the chart arising due to the subsequent blowing-up process. When the colliding brane approaches the stack of branes as $\mathrm{O}\left(s^{2}\right)$, we obtain additional conifold singularity at the enhanced point, which completes the full Dynkin diagram of the enhanced group as was found previously.

KEYwords: F-Theory, Differential and Algebraic Geometry

ArXiv EPRINT: 2003.05563 


\section{Contents}

1 Introduction 2

2 Half-hypermultiplets in six-dimensional F-theory 4

$\begin{array}{lll}3 & \mathrm{SO}(12) \rightarrow E_{7} & 7\end{array}$

3.1 Incomplete resolution: blowing up $p_{1}$ first 8

3.1.1 Blowing up process 8

3.1.2 Intersection patterns at $s \neq 0$ and $s=0 \quad 11$

3.1.3 Intersection diagram at $s=0$ : transmutation of a root into a weight 15

$\begin{array}{ll}3.2 \text { Complete resolution: blowing up } p_{1} \text { first } & 18\end{array}$

$\begin{array}{lll}3.2 .1 & \text { Blowing up process } & 18\end{array}$

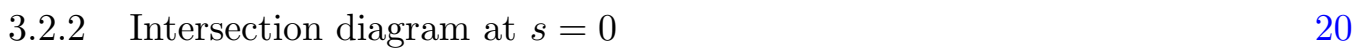

3.3 Incomplete resolution: blowing up $q_{1}$ first 22

$\begin{array}{lll}3.3 .1 & \text { Blowing up process } & 22\end{array}$

3.3.2 Intersection diagram at $s=0$ : differences from the $p_{1}$-first case $\quad 24$

3.4 Complete resolutions: blowing up $q_{1}$ first 25

3.5 Other inequivalent orderings 26

3.6 Comparison with the results of the M-theory Coulomb branch analysis 28

$4 \quad E_{7} \rightarrow E_{8} \quad 29$

4.1 Incomplete resolution: blowing up $p_{2}$ first 29

$\begin{array}{lll}\text { 4.1.1 } & \text { Blowing up process } & 29\end{array}$

4.1.2 Intersection diagram at $s=0 \quad 33$

$\begin{array}{lll}4.2 & \text { Complete resolution } & 35\end{array}$

4.3 Incomplete/complete resolutions: blowing up $q_{2}$ first 36

$\begin{array}{lll}4.4 & \text { Other inequivalent orderings } & 37\end{array}$

5 Conclusions $\quad 40$

A Symplectic Majorana-Weyl spinors, pseudo-real representations and half$\begin{array}{ll}\text { hypermultiplets } & 41\end{array}$

A.1 Symplectic Majorana-Weyl spinors 41

A.2 Pseudo-real representation and symplectic Majorana condition 42

A.3 $\frac{\mathbf{2 n}}{2}$ hypermultiplets vs. 2n $\frac{1}{2}$ hypermultiplets 43

A.4 Restriction on the complex scalars 45

B Summary of SU(6) $\rightarrow E_{6}$

$\begin{array}{ll}\text { C Small resolution of a conifold singularity } & 47\end{array}$ 


\section{Introduction}

The significance of F-theory [1] in modern particle physics model building cannot be overestimated. The characteristic features of the Standard Model can be naturally explained by the $\mathrm{SU}(5)$ and $\mathrm{SO}(10)$ grand unified theories, which are engineered in F-theory. It can achieve matter fields in the spinor representation as well as the exceptional group gauge symmetry. F-theory has an advantage over the $E_{8} \times E_{8}$ heterotic string theory in that it can address the issue of the gauge/gravity coupling correlation in the latter [2]. F-theory can also generate up-type Yukawa couplings perturbatively forbidden in D-brane models [3, 4].

In F-theory, matter typically arises ${ }^{1}$ at codimension-two singularities [5-9] in the base space of the elliptic fibration. To be specific, consider a six-dimensional F-theory compactified on an elliptically fibered three-fold defined by a hypersurface $\Phi(x, y, z, s)=0$ in $\mathbb{C}^{4} \ni(x, y, z, s)$ over a complex two-fold with local coordinates $(z, s) \in \mathbb{C}^{2}$. Over a generic point with fixed $(z, s)$, this equation describes a genus-one curve. We assume that this hypersurface has a codimension-one singularity of some compact group $H$ along $z=0$ for generic $s \neq 0$, which is enhanced to some compact group $G \supset H$ at a codimension-two particular locus along the line $z=0$, say, at $s=0$.

In this set-up, one typically obtains chiral matter hypermultiplets corresponding to the homogeneous Kähler manifold $G /(H \times \mathrm{U}(1))[5-10]$ at the enhanced singularity. However, if $G$ and $H$ are such that

$$
\begin{aligned}
G & \supset H \times \mathrm{SU}(2) \\
\operatorname{dim} \boldsymbol{G} & =(\operatorname{dim} \boldsymbol{H}, \mathbf{1}) \oplus(\mathbf{2 n}, \mathbf{2}) \oplus(\mathbf{1}, \mathbf{3}),
\end{aligned}
$$

where $n=10,16,28$ for $(G, H)=\left(E_{6}, \mathrm{SU}(6)\right),\left(E_{7}, \mathrm{SO}(12)\right),\left(E_{8}, E_{7}\right)$, respectively, ${ }^{2}$ the matter arising there are not the full hypermultiplets but half-hypermultiplets for generic complex structures achieving such singularities.

In all the cases above, $\mathbf{2} \mathbf{n}$ is a pseudo-real representation of $H$, of which the representation matrix can be written as an $\operatorname{Sp}(2 n)$ matrix. For these representations, one can impose the symplectic Majorana condition on the $2 n$ complex spinors. One can also define a similar relation among $2 n$ pairs of the complex scalars by using a $\mathrm{U}(1)_{R}$ rotation. In this way, the degrees of freedom of hypermultiplets are halved, yielding half-hypermultiplets in these representations.

In [11], the resolution of a codimension-two enhancement from $\mathrm{SU}(6)$ to $E_{6}$ was studied in six dimensions. It was shown there that, if the colliding brane approaches $z=0$ like $\mathrm{O}(s)$ which is the generic case, only five exceptional curves appear through the blowing-up process even at the singularity enhanced point. This type of resolution was called the incomplete resolution in [11]. Note that, by resolving a codimension-one $E_{6}$ singularity, one obtains six exceptional fibers consisting the $I V^{*}$ fiber type of Kodaira.

\footnotetext{
${ }^{1}$ aside from adjoint matter in four dimensions arising from 7-branes wrapped over a four-cycle with nontrivial relevant cohomologies.

${ }^{2}$ They are also the pairs (though different real forms) appearing in "magical" supergravity theories $[12,13]$.
} 
This means that there are not enough new degrees of freedom arising at the enhanced point to generate matter in a full hypermultiplet. The five exceptional fibers form a curious non-Dynkin intersection diagram, however. In particular, some of the exceptional curves turn out to have self-intersection number $-\frac{3}{2}$. This is equal to the minus of the length squared of a weight of the $\mathbf{2 0}$ representation. One can verify that these five exceptional curves form extremal rays of the cone of the positive weights of the $\mathbf{2 0}$ representation. (Actually, one can show that there are just 20 integer linear combinations of these exceptional curves which have self-intersection number $-\frac{3}{2}$; half of them have positive coefficients and the other half have negative coefficients, giving a whole set of the weights of 20.) Thus the matter forms a single $\mathbf{2 0}$ representation of $\mathrm{SU}(6)$. This shows the mechanism of how the half-hypermultiplet appears in this codimension-two singularity enhancement. (Note that for a full hypermultiplet one needs two $\mathbf{2 0}$ representations, either one of which survives as massless matter in six dimensions.)

On the other hand, if the brane collides like $\mathrm{O}\left(s^{2}\right)$, an extra singularity arises at the intersection. This is a conifold singularity. Resolving it yields another exceptional curve, completing the proper Dynkin intersection diagram expected from the ordinary Kodaira classification. This was called the complete resolution [11].

Higher codimension singularities were also studied in [14] by exploring the phases of three-dimensional gauge theory arising from the compactification of M-theory on a CalabiYau four-fold (see [15-20]). In this approach, the generation of a half-hypermultiplet was explained as a result of the reduction of the massless states occurring due to the monodromy among the fibers [14]. It was noted there that if the complex structure was tuned so that there were extra sections, the monodromy was reduced and a full hypermultiplet appeared.

In this paper, we study the resolutions of the other two cases, $\mathrm{SO}(12) \rightarrow E_{7}$ and $E_{7} \rightarrow E_{8}$, of the codimension-two singularity enhancement in which half-hypermultiplets appear as massless states at the singularity. The strategy is the same as that used in [11]. To blow up this singularity along the line $z=0$ we replace the local ambient space $\mathbb{C}^{4}$ with

$$
\widehat{\mathbb{C}}^{4}=\left\{((x, y, z, s) ;(\xi: \eta: \zeta)) \in \mathbb{C}^{4} \times \mathbb{P}^{2} \mid x \eta-y \xi=y \zeta-z \eta=z \xi-x \zeta=0\right\}
$$

and consider the hypersurface in it. (1.2) inserts a continuous family of $\mathbb{P}^{2}$ along the complex line $z=0$ for arbitrary $s \in \mathbb{C}$. Then we find that there still are several, (again) codimensionone singularities on the intersection of $\widehat{\mathbb{C}}^{4}(1.2)$ and the hypersurface $\Phi=0$. To resolve these singularities, we further make a replacement similar to $\mathbb{C}^{4} \rightarrow \widehat{\mathbb{C}}^{4}$ along each singular line and consider the hypersurface in this blown-up ambient space. Then if we still find some singularities of codimension one, we perform a codimension-one blow up along each of them. Repeating these steps we end up with either of the two cases:

(1) The case where there are no more singularities of any kind on the final hypersurface.

(2) The case where there are no more codimension-one singularities, but still there is an isolated codimension-two singularity at $s=0$ on the final hypersurface.

This is the phenomenon known as the partial simultaneous resolution of singularities [14, 21-23], and which case we will end up with depends on the vanishing order of the 
relevant section of the projective-space bundle for the respective singularity enhancement. For the enhancement $\mathrm{SU}(6) \rightarrow E_{6}$ studied in [11], the relevant section is the polynomial $t_{r}(s)$ in eq. (2.6) (see below), for $\mathrm{SO}(12) \rightarrow E_{7}$ it is $H_{n+4}(s)$, and for $E_{7} \rightarrow E_{8}$ it is $f_{n+8}(s)$. We will show that, also for the cases $\mathrm{SO}(12) \rightarrow E_{7}$ and $E_{7} \rightarrow E_{8}$ we will consider in this paper, we are led to the result (1) if these sections vanish like $\mathrm{O}(s)$ as $s \rightarrow 0$. The codimension-two singularity is then resolved only by the codimension-one blow-ups in the ambient space, and therefore the number of times of the blowing-up operations is the same as that needed to resolve a Kodaira $H$ singularity for fixed generic $s \neq 0$. Thus there are not enough exceptional curves to form the proper Dynkin diagram of $G$ as their intersection matrix. Even so, we will see that the intersection diagram of the exceptional curves obtained by the $s \rightarrow 0$ limit of the exceptional surfaces is different from the Dynkin diagram of $H$. Rather, it turns out that some of the curves have their self-intersection number $-\frac{3}{2}$, as was observed in [11] for the $\mathrm{SU}(6) \rightarrow E_{6}$ enhancement.

We perform blowing-ups for all possible inequivalent orderings of blowing up the singularities in both the enhancements $\mathrm{SO}(12) \rightarrow E_{7}$ and $E_{7} \rightarrow E_{8}$. We find that the intersection diagram on every other row in figures 33 and 44 of [14] can be obtained, but not all of them. ${ }^{3}$ Although the intersection diagrams are different, the fibers obtained in the limit $s \rightarrow 0$ always form extremal rays of the cone of the positive weights of the relevant pseudo-real representation.

On the other hand, if they vanish like $\mathrm{O}\left(s^{2}\right)$, we are led to the result (2), where we need a further resolution of the isolated singularity. This is the complete resolution; we have one more additional node to the incomplete intersection diagram obtained in the incomplete resolution, obtaining the full Dynkin diagram of the group $G$. We will find that, as was observed in [11], this final singularity appearing at codimension two is a conifold singularity for all the cases we examine in this paper.

The plan of this paper is as follows. In section 2, we review in what circumstances massless matter fields appear as half-hypermultiplets in the global six-dimensional F-theory compactification on an elliptic Calabi-Yau three-fold over a Hirzebruch surface. In section 3, we present the detailed process of blow-ups for the codimension-two singularity enhancement from $\mathrm{SO}(12)$ to $E_{7}$. In section 4, we turn to the resolution of the enhancement from $E_{7}$ to $E_{8}$. Section 5 summarizes the conclusions. In appendix A, we explain the relations between symplectic Majorana-Weyl spinors, pseudo-real representations and half-hypermultiplets. In appendix B, we summarize the results of [11] on the resolution of the enhancement $\mathrm{SU}(6) \rightarrow E_{6}$. Finally, in appendix C, we present a basic explanation of the small resolution of a conifold.

\section{Half-hypermultiplets in six-dimensional F-theory}

Half-hypermultiplets arise when the unbroken gauge group is $\mathrm{SU}(6), \mathrm{SO}(12)$ or $E_{7}$ [7]. These models can be systematically obtained by tuning the complex structure of the $\mathrm{SU}(5)$ model.

\footnotetext{
${ }^{3}$ In the enhancement $\mathrm{SU}(6) \rightarrow E_{6}$ studied in [11], there is no such option since only one singularity appears at each step of blowing up.
} 
We start with the six-dimensional compactification on F-theory on an elliptically fibered Calabi-Yau three-fold over a Hirzebruch surface $\mathbb{F}_{n}[5,6]$. Let $z, z^{\prime}$ be affine coordinates of the fiber and base $\mathbb{P}^{1}$ 's, respectively. The Weierstrass model

$$
y^{2}=x^{3}+f\left(z, z^{\prime}\right) x+g\left(z, z^{\prime}\right)
$$

develops an $\mathrm{SU}(5)$ singularity if [7]

$$
\begin{aligned}
f\left(z, z^{\prime}\right)= & -3 h_{n+2}^{4}+12 h_{n+2}^{2} H_{n+4} z-12\left(H_{n+4}^{2}-h_{n+2} q_{n+6}\right) z^{2}+f_{n+8} z^{3}+f_{8} z^{4}, \\
g\left(z, z^{\prime}\right)= & 2 h_{n+2}^{6}-12 h_{n+2}^{4} H_{n+4} z+\left(24 h_{n+2}^{2} H_{n+4}^{2}-12 h_{n+2}^{3} q_{n+6}\right) z^{2} \\
& +\left(-f_{n+8} h_{n+2}^{2}+24 h_{n+2} H_{n+4} q_{n+6}-16 H_{n+4}^{3}\right) z^{3} \\
& +\left(-f_{8} h_{n+2}^{2}+2 f_{n+8} H_{n+4}+12 q_{n+6}^{2}\right) z^{4}+g_{n+12} z^{5}+g_{12} z^{6},
\end{aligned}
$$

where $h_{n+2}, H_{n+4}, q_{n+6}, f_{n+8}$ and $g_{n+12}$ are polynomials of $z^{\prime}$ of degrees specified by the subscripts. They are sections of Looijenga's weighted projective space bundle [24-26] char-

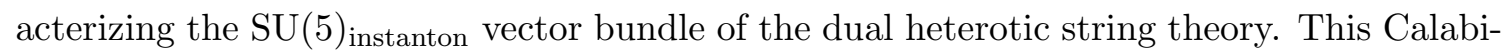
Yau three-fold admits a $K 3$ fibration, and we work with one of the rational elliptic surfaces in the stable degeneration limit of the $K 3$ so that the orders of the polynomials $f\left(z, z^{\prime}\right)$ and $g\left(z, z^{\prime}\right)$ are truncated at $z^{4}$ and $z^{6}$, respectively. This suffices since the anomalies cancel for each $E_{8}$ gauge group, and also we are interested in the local structure of the singularity. $x$ and $y$ are then taken to be sections of $\mathcal{O}\left(2\left(-K_{\mathbb{F}_{n}}-C_{0}\right)\right)$ and $\mathcal{O}\left(3\left(-K_{\mathbb{F}_{n}}-C_{0}\right)\right)$, where $C_{0}$ is a divisor class with $C_{0}^{2}=-n$, satisfying $-K_{\mathbb{F}_{n}}=2 C_{0}+(2+n) f$ with the fiber class $f$. Similar modifications are necessary for $f\left(z, z^{\prime}\right)$ and $g\left(z, z^{\prime}\right)$. This deviation from the anti-canonical class (and hence from a Calabi-Yau) is because we consider a rational-elliptic-surface fibration.

The Weierstrass equation (2.1) with (2.2) can be written in Tate's form as

$$
y^{\prime 2}+x^{\prime 3}+\alpha_{4} z^{4} x^{\prime}+\alpha_{6} z^{6}+a_{0} z^{5}+a_{2} z^{3} x^{\prime}+a_{3} z^{2} y^{\prime}+a_{4} z x^{\prime 2}+a_{5} x^{\prime} y^{\prime}=0
$$

with

$$
\begin{aligned}
& a_{5}=2 \sqrt{3} i h_{n+2}, \\
& a_{4}=-6 H_{n+4}, \\
& a_{3}=4 \sqrt{3} i q_{n+6}, \\
& a_{2}=f_{n+8}, \\
& a_{0}=g_{n+12}-2 H_{n+4} f_{8}, \\
& \alpha_{4}=f_{8}, \\
& \alpha_{6}=g_{12} .
\end{aligned}
$$

For completeness we write $x, y$ in (2.1) in terms of $x^{\prime}, y^{\prime}$ in (2.3):

$$
\begin{aligned}
& x=x^{\prime}+\frac{1}{3}\left(a_{4} z-\frac{1}{4} a_{5}^{2}\right), \\
& y=i\left(y^{\prime}+\frac{1}{2}\left(a_{5} x^{\prime}+a_{3} z^{2}\right)\right) .
\end{aligned}
$$


SU(6). To obtain an equation for SU(6) gauge group, which yields half-hypermultiplets, we set $[7]$

$$
\begin{aligned}
h_{n+2} & =t_{r} h_{n+2-r}, \\
H_{n+4} & =t_{r} H_{n+4-r}, \\
q_{n+6} & =u_{r+4} h_{n+2-r}, \\
f_{n+8} & =t_{r} f_{n+8-r}-12 u_{r+4} H_{n+4-r}, \\
g_{n+12} & =2\left(u_{r+4} f_{n-r+8}+f_{8} t_{r} H_{n-r+4}\right) .
\end{aligned}
$$

Then the spectral cover factorizes as

$$
\begin{aligned}
0 & =a_{0} z^{5}+a_{2} z^{3} x^{\prime}+a_{3} z^{2} y^{\prime}+a_{4} z x^{\prime 2}+a_{5} x^{\prime} y^{\prime} \\
& =\left(x^{\prime} t_{r}+2 z^{2} u_{r+4}\right)\left(z^{3} f_{n-r+8}+2 i \sqrt{3} y^{\prime} h_{n-r+2}-6 z x^{\prime} H_{n-r+4}\right),
\end{aligned}
$$

indicating that the $\mathrm{SU}(5)$ instanton is reduced to an $\mathrm{SU}(3) \times \mathrm{SU}(2)$ instanton in the heterotic dual, at the same time the Mordell-Weil rank of the rational elliptic surface is reduced. The Mordell-Weil lattice is No.15 in the Oguiso-Shioda classification [27]. In this specification $f\left(z, z^{\prime}\right)$ and $g\left(z, z^{\prime}\right)$ become

$$
\begin{aligned}
f_{\mathrm{SU}(6)}\left(z, z^{\prime}\right)= & -3 t_{r}^{4} h_{n-r+2}^{4}+12 z t_{r}^{3} h_{n-r+2}^{2} H_{n-r+4}+z^{2}\left(12 t_{r} u_{r+4} h_{n-r+2}^{2}-12 t_{r}^{2} H_{n-r+4}^{2}\right) \\
& +z^{3}\left(t_{r} f_{n-r+8}-12 u_{r+4} H_{n-r+4}\right)+f_{8} z^{4}, \\
g_{\mathrm{SU}(6)}\left(z, z^{\prime}\right)= & 2 t_{r}^{6} h_{n-r+2}^{6}-12 z t_{r}^{5} h_{n-r+2}^{4} H_{n-r+4} \\
& +z^{2}\left(24 t_{r}^{4} h_{n-r+2}^{2} H_{n-r+4}^{2}-12 t_{r}^{3} u_{r+4} h_{n-r+2}^{4}\right) \\
& +z^{3}\left(-t_{r}^{3} f_{n-r+8} h_{n-r+2}^{2}+36 t_{r}^{2} u_{r+4} h_{n-r+2}^{2} H_{n-r+4}-16 t_{r}^{3} H_{n-r+4}^{3}\right) \\
& +z^{4}\left(-f_{8} t_{r}^{2} h_{n-r+2}^{2}+2 t_{r}^{2} f_{n-r+8} H_{n-r+4}+12 u_{r+4}^{2} h_{n-r+2}^{2}-24 t_{r} u_{r+4} H_{n-r+4}^{2}\right) \\
& +z^{5}\left(2 f_{8} t_{r} H_{n-r+4}+2 u_{r+4} f_{n-r+8}\right)+g_{12} z^{6} .
\end{aligned}
$$

The discriminant reads

$$
\begin{aligned}
\Delta_{\mathrm{SU}(6)}= & 9 z^{6} t_{r}^{3} h_{n-r+2}^{4}\left[t_{r}^{3}\left(12 g_{12} h_{n-r+2}^{2}-f_{n-r+8}^{2}\right)\right. \\
& +t_{r}^{2}\left(-24 f_{8} u_{r+4} h_{n-r+2}^{2}-24 u_{r+4} f_{n-r+8} H_{n-r+4}\right) \\
& \left.-144 t_{r} u_{r+4}^{2} H_{n-r+4}^{2}-96 u_{r+4}^{3} h_{n-r+2}^{2}\right]+O\left(z^{7}\right) .
\end{aligned}
$$

Thus the Weierstrass model with (2.8), (2.9) indeed has a codimension-one SU(6) singularity along $z=0$.

The zero loci of $t_{r}$ are the points where the $\mathrm{SU}(6)$ singularity is enhanced to $E_{6}$, those of $h_{n-r+2}$ are the ones to $D_{6}$, and those of the remaining factor of degree $2 n+r+16$ are the ones to $A_{6}$. They respectively yield $r$ half-hypermultiplets in $\mathbf{2 0}, n-r+2$ hypermultiplets in $\mathbf{1 5}$ and $2 n+r+16$ hypermultiplets in $\mathbf{6}$.

The number of the complex structure moduli is $3 n-r+21$, which satisfies the anomalyfree constraint for one of the $E_{8}$ factors [7]

$$
\begin{aligned}
n_{H}-n_{V} & =20 \cdot \frac{r}{2}+15(n-r+2)+6(2 n+r+16)+3 n-r+21-35 \\
& =30 n+112 .
\end{aligned}
$$

Note that this condition does not hold if the multiplets in $\mathbf{2 0}$ are ordinary hypermultiplets. 
SO(12). To further obtain an equation for $\mathrm{SO}(12)$ gauge group, one only needs to set $h_{n+2-r}=0$ in (2.6). The spectral cover is now

$$
\left(x^{\prime} t_{r}+2 z^{2} u_{r+4}\right)\left(x^{\prime} H_{n-r+4}-\frac{1}{6} z^{2} f_{n-r+8}\right)=0 .
$$

These factors are in the same form, corresponding to two $\mathrm{SU}(2)$ 's of the instanton gauge group of the heterotic theory. The Mordell-Weil lattice is No.26 in [27].

Then $f\left(z, z^{\prime}\right)$ and $g\left(z, z^{\prime}\right)$ are

$$
\begin{aligned}
f_{\mathrm{SO}(12)}\left(z, z^{\prime}\right)= & -12 z^{2} t_{r}^{2} H_{n-r+4}^{2}+z^{3}\left(t_{r} f_{n-r+8}-12 u_{r+4} H_{n-r+4}\right)+f_{8} z^{4}, \\
g_{\mathrm{SO}(12)}\left(z, z^{\prime}\right)= & -16 z^{3} t_{r}^{3} H_{n-r+4}^{3}+2 z^{4}\left(t_{r}^{2} f_{n-r+8} H_{n-r+4}-12 t_{r} u_{r+4} H_{n-r+4}^{2}\right) \\
& +2 z^{5}\left(f_{8} t_{r} H_{n-r+4}+u_{r+4} f_{n-r+8}\right)+g_{12} z^{6} .
\end{aligned}
$$

The discriminant is

$$
\Delta_{\mathrm{SO}(12)}=-36 z^{8} t_{r}^{2} H_{n-r+4}^{2}\left(t_{r} f_{n-r+8}+12 u_{r+4} H_{n-r+4}\right)^{2}+O\left(z^{9}\right) .
$$

The zero loci of both $t_{r}$ and $H_{n-r+4}$ give rise to $E_{7}$ singularities to yield $n+4$ halfhypermultiplets. The loci of the remaining factor are $A_{7}$ singularities, giving $n+8$ hypers in 12. With additional neutral hypermultiplets from the $2 n+18$ complex structure moduli, we have

$$
\begin{aligned}
n_{H}-n_{V} & =32 \cdot \frac{n+4}{2}+12(n+8)+2 n+18-66 \\
& =30 n+112
\end{aligned}
$$

as it should be. Again, if $\mathbf{3 2}$ is not a half-hyper, the anomaly does not cancel.

$\boldsymbol{E}_{\mathbf{7}}$. Finally, the $E_{7}$ model can be obtained by setting $h_{n+2}=H_{n+4}=q_{n+6}=0$ in the $\mathrm{SU}(5)$ model. The gauge group of the heterotic vector bundle is $\mathrm{SU}(2)$. The Mordell-Weil lattice is No.43 in [27]. $f\left(z, z^{\prime}\right)$ and $g\left(z, z^{\prime}\right)$ are simply given by

$$
\begin{aligned}
& f_{E_{7}}\left(z, z^{\prime}\right)=f_{n+8} z^{3}+f_{8} z^{4}, \\
& g_{E_{7}}\left(z, z^{\prime}\right)=g_{n+12} z^{5}+g_{12} z^{6} .
\end{aligned}
$$

The discriminant

$$
\Delta_{E_{7}}=4 f_{n+8}^{3} z^{9}+O\left(z^{10}\right)
$$

implies that $n+8$ half-hypermultiplets in $\mathbf{5 6}$ of $E_{7}$ arise. Again they must be half-hyper as

$$
\begin{aligned}
n_{H}-n_{V} & =56 \cdot \frac{n+8}{2}+2 n+21-133 \\
& =30 n+112 .
\end{aligned}
$$

\section{$3 \quad \mathrm{SO}(12) \rightarrow E_{7}$}

We consider a Weierstrass model on a base two-fold $B_{2}$ with local coordinates $\{z, s\}$, where the codimension-one singularity arises along $z=0$ and the codimension-two singularity arises at $s=0$ on the $z=0$ complex line. $s$ corresponds to $z^{\prime}$ in the previous section. 


\subsection{Incomplete resolution: blowing up $p_{1}$ first}

\subsubsection{Blowing up process}

We consider a concrete Weierstrass model of incomplete resolution by setting ${ }^{4} H_{n-r+4}=s$, $t_{r}=-\frac{1}{2}, f_{n-r+8}=-2$ and $u_{r+4}=f_{8}=g_{12}=0$ in (2.13) and (2.14):

$$
\Phi(x, y, z, s)=-y^{2}+x^{3}+f(z, s) x+g(z, s)=0,
$$

where

$$
\begin{aligned}
& f(z, s)=-3 s^{2} z^{2}+z^{3}, \\
& g(z, s)=2 s^{3} z^{3}-s z^{4} .
\end{aligned}
$$

At $s \neq 0$, the orders of $f, g$ and the discriminant $\Delta$ in $z$ are $(2,3,8)$, while at $s=0$, they satisfy $(3, \geq 5,9)$. Therefore $(3.2)$ describes the enhancement $I_{2}^{*} \rightarrow I I I^{*}\left(\mathrm{SO}(12) \rightarrow E_{7}\right)$ of the Kodaira type, satisfying the requirement.

1st blow up. With (3.2), the equation (3.1) reads

$$
\Phi(x, y, z, s)=x z^{2}\left(z-3 s^{2}\right)+s z^{3}\left(2 s^{2}-z\right)+x^{3}-y^{2}=0 .
$$

Eq. (3.3) has a codimension-one singularity at $p_{0} \equiv(0,0,0, s)$. We blow up this by replacing the complex line $(x, y, z)=(0,0,0)$ with $\mathbb{P}^{2} \times \mathbb{C}$ in $\mathbb{C}^{4}$ by passing to the following charts corresponding to three affine patches of $\mathbb{P}^{2}$ for fixed $s$ :

\section{Chart $1_{x}$}

$$
\begin{aligned}
\Phi\left(x, x y_{1}, x z_{1}, s\right) & =x^{2} \Phi_{x}\left(x, y_{1}, z_{1}, s\right) \\
\Phi_{x}\left(x, y_{1}, z_{1}, s\right) & =x^{2}\left(z_{1}^{3}-s z_{1}^{4}\right)+x\left(s z_{1}-1\right)^{2}\left(2 s z_{1}+1\right)-y_{1}^{2} . \\
\mathcal{C}_{p_{0}} \text { in } 1_{x} & : x=0, y_{1}=0 . \\
\text { Singularities } & :\left(x, y_{1}, z_{1}, s\right)=\left(0,0, \frac{1}{s}, s\right),\left(0,0,-\frac{1}{2 s}, s\right) .
\end{aligned}
$$

These singularities are of codimension one, which we refer to as $p_{1}$ and $q_{1}$, respectively. $\mathcal{C}_{p_{0}}$ is the exceptional curve at fixed $s$.

\section{Chart $1_{y}$}

$$
\begin{aligned}
\Phi\left(x_{1} y, y, y z_{1}, s\right) & =y^{2} \Phi_{y}\left(x_{1}, y, z_{1}, s\right) \\
\Phi_{y}\left(x_{1}, y, z_{1}, s\right) & =2 s^{3} y z_{1}^{3}+x_{1} y z_{1}^{2}\left(y z_{1}-3 s^{2}\right)-s y^{2} z_{1}^{4}+x_{1}^{3} y-1 . \\
\mathcal{C}_{p_{0}} \text { in } 1_{y} & : \text { Invisible in this patch. } \\
\text { Singularities } & : \text { None. }
\end{aligned}
$$

In chart $1_{y}$, the exceptional curve cannot be seen and there is no singularity.

\footnotetext{
${ }^{4}$ The same model is obtained by setting $t_{r}=s, H_{n-r+4}=-\frac{1}{2}, u_{r+4}=\frac{1}{6}$ and $f_{n-r+8}=f_{8}=g_{12}=0$.
} 
Chart $1_{z}$

$$
\begin{aligned}
\Phi\left(x_{1} z, y_{1} z, z, s\right) & =z^{2} \Phi_{z}\left(x_{1}, y_{1}, z, s\right) \\
\Phi_{z}\left(x_{1}, y_{1}, z, s\right) & =z\left(2 s^{3}-3 s^{2} x_{1}-s z+x_{1}^{3}+x_{1} z\right)-y_{1}^{2} . \\
\mathcal{C}_{p_{0}} \text { in } 1_{z} & : z=0, y_{1}=0 . \\
\text { Singularities } & :\left(x_{1}, y_{1}, z, s\right)=(s, 0,0, s),(-2 s, 0,0, s) .
\end{aligned}
$$

The first singularity is $p_{1}$, while the second is $q_{1}$.

2nd blow up. By the 1st blow up we found two singularities. There are two ways to resolve them; either we blow up $p_{1}$ first, or $q_{1}$ first. In this section we blow up $p_{1}$ first.

In order to blow up the singularity $p_{1}$ in $\Phi_{z}\left(x_{1}, y_{1}, z, s\right)=0$, we shift the coordinate $x_{1}$ so that the position of the singularity becomes $(0,0,0, s)$ :

$$
\Psi_{z}\left(\tilde{x}_{1}, y_{1}, z, s\right) \equiv \Phi_{z}\left(\tilde{x}_{1}+s, y_{1}, z, s\right) .
$$

The singularities of $\Psi_{z}\left(\tilde{x}_{1}, y_{1}, z, s\right)=0$ are now at $(0,0,0, s)\left(=p_{1}\right)$ and $(-3 s, 0,0, s)\left(=q_{1}\right)$. We blow up the singularity of $\Psi_{z}\left(\tilde{x}_{1}, y_{1}, z, s\right)=0$ at $(0,0,0, s)$.

Chart $2_{z x}$

$$
\begin{aligned}
\Psi_{z}\left(\tilde{x}_{1}, \tilde{x}_{1} y_{2}, \tilde{x}_{1} z_{2}, s\right)= & \tilde{x}_{1}^{2} \Psi_{z x}\left(\tilde{x}_{1}, y_{2}, z_{2}, s\right), \\
\Psi_{z x}\left(\tilde{x}_{1}, y_{2}, z_{2}, s\right)= & \tilde{x}_{1} z_{2}\left(3 s+\tilde{x}_{1}+z_{2}\right)-y_{2}^{2} . \\
\mathcal{C}_{p_{1}} \text { in } 2_{z x}: & \tilde{x}_{1}=0, \quad y_{2}=0 . \\
\text { Singularities }: & \left(\tilde{x}_{1}, y_{2}, z_{2}, s\right)=(0,0,0, s)\left(=p_{2}\right),(0,0,-3 s, s)\left(=r_{2}\right), \\
& (-3 s, 0,0, s)\left(=q_{1}\right) .
\end{aligned}
$$

We find three singularities in this chart and name them as shown in the parentheses.

Chart $2_{z y}$ In this chart, we find no singularity so we omit the details of $\Psi_{z y}$.

Chart $2_{z z}$

$$
\begin{aligned}
\Psi_{z}\left(x_{2} z, y_{2} z, z, s\right) & =z^{2} \Psi_{z z}\left(x_{2}, y_{2}, z, s\right) \\
\Psi_{z z}\left(x_{2}, y_{2}, z, s\right) & =x_{2} z\left(3 s x_{2}+x_{2}^{2} z+1\right)-y_{2}^{2} . \\
\mathcal{C}_{p_{1}} \text { in } 2_{z z} & : z=0, \quad y_{2}=0 . \\
\text { Singularities } & :\left(x_{2}, y_{2}, z, s\right)=(0,0,0, s)\left(=q_{2}\right),\left(-\frac{1}{3 s}, 0,0, s\right)\left(=r_{2}\right) .
\end{aligned}
$$

We observe two singularities. The former $\left(q_{2}\right)$ is one which can only be seen in this chart, while the latter $\left(r_{2}\right)$ is already seen in chart $2_{z x}$.

Here the process branches off in three ways depending on which of the three singularities $p_{2}, r_{2}$ and $q_{1}$ in chart $2_{z x}$ is blown up next. Although they are separated for $s \neq 0$, they coincide with each other at $s=0$ (see (3.8)). For this reason, changing the order of blowing up these three singularities changes the subsequent geometries. In this section, we consider the case $p_{2}$ is blown up next. On the other hand, $q_{2}$ in chart $2_{z z}$ is separated with the other three singularities even at $s=0$, and hence it can be independently blown up. We leave the blow-up of $q_{2}$ until later and proceed with blowing up $p_{2}$. 
3rd blow up at $\boldsymbol{p}_{\mathbf{2}}$. We blow up $p_{2}$ in chart $2_{z x}$ :

Chart $3_{z x x}$

$$
\begin{aligned}
\Psi_{z x}\left(\tilde{x}_{1}, \tilde{x}_{1} y_{3}, \tilde{x}_{1} z_{3}, s\right) & =\tilde{x}_{1}^{2} \Psi_{z x x}\left(\tilde{x}_{1}, y_{3}, z_{3}, s\right) \\
\Psi_{z x x}\left(\tilde{x}_{1}, y_{3}, z_{3}, s\right) & =z_{3}\left(3 s+\tilde{x}_{1} z_{3}+\tilde{x}_{1}\right)-y_{3}^{2} . \\
\mathcal{C}_{p_{2}} \text { in } 3_{z x x} & : \tilde{x}_{1}=0, y_{3}^{2}=3 s z_{3} . \\
\text { Singularities } & :\left(\tilde{x}_{1}, y_{3}, z_{3}, s\right)=(-3 s, 0,0, s)\left(=q_{1}\right) .
\end{aligned}
$$

Chart $3_{z x y}$ Regular.

Chart $3_{z x z}$

$$
\begin{aligned}
\Psi_{z x}\left(x_{3} z_{2}, y_{3} z_{2}, z_{2}, s\right) & =z_{2}^{2} \Psi_{z x z}\left(x_{3}, y_{3}, z_{2}, s\right), \\
\Psi_{z x z}\left(x_{3}, y_{3}, z_{2}, s\right) & =3 s x_{3}+x_{3}\left(x_{3}+1\right) z_{2}-y_{3}^{2} . \\
\mathcal{C}_{p_{2}} \text { in } 3_{z x z} & : z_{2}=0, y_{3}^{2}=3 s x_{3} . \\
\text { Singularities } & :\left(x_{3}, y_{3}, z_{2}, s\right)=(0,0,-3 s, s)\left(=r_{2}\right) .
\end{aligned}
$$

The remaining singularities are resolved by blowing up $q_{1}, r_{2}$ and $q_{2}$, which are all codimension one. $q_{1}$ and $r_{2}$, which were overlapping at $s=0$ in chart $2_{z x}$ before blowing up $p_{2}$, are now contained in different charts $3_{z x x}$ and $3_{z x z}$, respectively, and are separated even at $s=0$. Also, $q_{2}$ stays in chart $2_{z z}$ and never coincides with them. Therefore, the remaining three singularities are all separated with each other for any $s$ and can be independently blown up. The procedure is similar as before and is easily done, but for later use, we complete the process here and present the relevant results. After blowing up $q_{1}$ in chart $3_{z x x}$ and $r_{2}$ in chart $3_{z x z}$, we return to chart $2_{z z}$ and blow up $q_{2}$. These blow-ups do not cause any new singularities.

4th blow up at $\boldsymbol{q}_{\mathbf{1}}$. To blow up $q_{1}$ in chart $3_{z x x}$, we shift the $\tilde{x}_{1}$ coordinate so that $q_{1}$ is at the origin:

$$
\Sigma_{z x x}\left(\tilde{\tilde{x}}_{1}, y_{3}, z_{3}, s\right) \equiv \Psi_{z x x}\left(\tilde{\tilde{x}}_{1}-3 s, y_{3}, z_{3}, s\right)
$$

Chart $4_{z x x x}$

$$
\begin{aligned}
\Sigma_{z x x}\left(\tilde{\tilde{x}}_{1}, \tilde{\tilde{x}}_{1} y_{4}, \tilde{\tilde{x}}_{1} z_{4}, s\right) & =\tilde{\tilde{x}}_{1}^{2} \Sigma_{z x x x}\left(\tilde{\tilde{x}}_{1}, y_{4}, z_{4}, s\right), \\
\Sigma_{z x x x}\left(\tilde{\tilde{x}}_{1}, y_{4}, z_{4}, s\right) & =-y_{4}^{2}+z_{4}\left(1+\tilde{\tilde{x}}_{1} z_{4}-3 z_{4} s\right) . \\
\mathcal{C}_{q_{1}} \text { in } 4_{z x x x} & : \tilde{\tilde{x}}_{1}=0, \quad y_{4}^{2}=z_{4}\left(1-3 z_{4} s\right) .
\end{aligned}
$$

Chart $4_{z x x y}$ We omit the details.

Chart $4_{z x x z}$

$$
\begin{aligned}
\Sigma_{z x x}\left(x_{4} z_{3}, y_{4} z_{3}, z_{3}, s\right) & =z_{3}^{2} \Sigma_{z x x z}\left(x_{4}, y_{4}, z_{3}, s\right), \\
\Sigma_{z x x z}\left(x_{4}, y_{4}, z_{3}, s\right) & =-y_{4}^{2}+x_{4}\left(1+z_{3}\right)-3 s . \\
\mathcal{C}_{q_{1}} \text { in } 4_{z x x z} & : z_{3}=0, \quad y_{4}^{2}=x_{4}-3 s .
\end{aligned}
$$


4th blow up at $\boldsymbol{r}_{\mathbf{2}}$. To blow up $r_{2}$ in chart $3_{z x z}$, we shift the $z_{2}$ coordinate so that $r_{2}$ is at the origin:

$$
\Sigma_{z x z}\left(x_{3}, y_{3}, \tilde{z}_{2}, s\right) \equiv \Psi_{z x z}\left(x_{3}, y_{3}, \tilde{z}_{2}-3 s, s\right)
$$

Chart $4_{z x z x}$

$$
\begin{aligned}
\Sigma_{z x z}\left(x_{3}, x_{3} y_{4}, x_{3} z_{4}, s\right) & =x_{3}^{2} \Sigma_{z x z x}\left(x_{3}, y_{4}, z_{4}, s\right), \\
\Sigma_{z x z x}\left(x_{3}, y_{4}, z_{4}, s\right) & =-y_{4}^{2}+z_{4}\left(1+x_{3}\right)-3 s . \\
\mathcal{C}_{r_{2}} \text { in } 4_{z x z x} & : x_{3}=0, y_{4}^{2}=z_{4}-3 s .
\end{aligned}
$$

Chart $4_{z x z y}$ We omit the details.

Chart $4_{z x z z}$

$$
\begin{aligned}
\Sigma_{z x z}\left(x_{4} \tilde{z}_{2}, y_{4} \tilde{z}_{2}, \tilde{z}_{2}, s\right) & =\tilde{z}_{2}^{2} \Sigma_{z x z z}\left(x_{4}, y_{4}, \tilde{z}_{2}, s\right) \\
\Sigma_{z x z z}\left(x_{4}, y_{4}, \tilde{z}_{2}, s\right) & =-y_{4}^{2}+x_{4}+x_{4}^{2}\left(\tilde{z}_{2}-3 s\right) . \\
\mathcal{C}_{r_{2}} \text { in } 4_{z x z z} & : \tilde{z}_{2}=0, \quad y_{4}^{2}=x_{4}-3 x_{4}^{2} s .
\end{aligned}
$$

3rd blow up at $\boldsymbol{q}_{\mathbf{2}}$. Finally, we go back to chart $2_{z z}$ and blow up $q_{2}(3.9)$ :

Chart $3_{z z x}$

$$
\begin{aligned}
\Psi_{z z}\left(x_{2}, x_{2} y_{3}, x_{2} z_{3}, s\right) & =x_{2}^{2} \Psi_{z z x}\left(x_{2}, y_{3}, z_{3}, s\right) \\
\Psi_{z z x}\left(x_{2}, y_{3}, z_{3}, s\right) & =-y_{3}^{2}+z_{3}\left(1+x_{2}^{3} z_{3}+3 x_{2} s\right) . \\
\mathcal{C}_{q_{2}} \text { in } 3_{z z x} & : x_{2}=0, \quad y_{3}^{2}=z_{3} .
\end{aligned}
$$

Chart $3_{z z y} \quad$ We omit the details.

Chart $3_{z z z}$

$$
\begin{aligned}
\Psi_{z z}\left(x_{3} z, y_{3} z, z, s\right) & =z^{2} \Psi_{z z z}\left(x_{3}, y_{3}, z, s\right), \\
\Psi_{z z z}\left(x_{3}, y_{3}, z, s\right) & =-y_{3}^{2}+x_{3}+x_{3}^{3} z^{3}+3 x_{3}^{2} z s . \\
\mathcal{C}_{q_{2}} \text { in } 3_{z z z} & : z=0, \quad y_{3}^{2}=x_{3} .
\end{aligned}
$$

The whole process of blowing up is summarized in table 1 .

\subsubsection{Intersection patterns at $s \neq 0$ and $s=0$}

Through the blowing up process, we have obtained six exceptional curves $\mathcal{C}_{I}\left(I \in\left\{p_{0}, p_{1}\right.\right.$, $\left.\left.p_{2}, q_{1}, r_{2}, q_{2}\right\}\right)$ at general $s$. For given $\mathcal{C}$, we define the corresponding exceptional curve $\delta$ as the $s \rightarrow 0$ limit of $\mathcal{C}$ in the chart where $\mathcal{C}$ originally is defined. One can then show that the intersection pattern of $\left\{\mathcal{C}_{I}\right\}$ at $s \neq 0$ and that of $\left\{\delta_{I}\right\}$ at $s=0$ are $D_{6}$ and $A_{6}$, respectively.

Let us present the detail of the derivation. Suppose $\mathcal{C}$ and $\delta$ arise from some blow-up and are defined in chart $A$, while $\mathcal{C}^{\prime}$ and $\delta^{\prime}$ arise from a subsequent blow-up and are defined in chart $A^{\prime}$. In order to see how $\mathcal{C}$ and $\delta$ intersect with $\mathcal{C}^{\prime}$ and $\delta^{\prime}$, one has to locate their positions in the same chart. This is done by lifting up $\mathcal{C}$ and $\delta$ from chart $A$ to chart $A^{\prime}$. 


\begin{tabular}{|c|l|l|l|l|}
\hline \multirow{2}{*}{$p_{0} \rightarrow$} & 1st blow up & 2nd blow up & 3rd blow up & 4th blow up \\
\cline { 3 - 4 }$(s: 0: 1) \rightarrow$ & $p_{2}(1: 0: 0)\left(\right.$ in $\left.2_{z x}\right) \rightarrow$ & regular & \\
& $p_{1}(-2 s: 0: 1)$ & $q_{1}(1: 0: 0)\left(\tilde{x}_{1}=-3 s\right)$ & $\mathscr{q}_{1}(1: 0: 0)\left(\tilde{x}_{1}=-3 s\right) \rightarrow$ & regular \\
& & $r_{2}(1: 0:-3 s)$ & $r_{2}(0: 0: 1)\left(z_{2}=-3 s\right) \rightarrow$ & regular \\
& & $q_{2}(0: 0: 1)\left(\right.$ in $\left.2_{z z}\right) \rightarrow$ & regular & \\
\hline
\end{tabular}

Table 1. $\mathrm{SO}(12) \rightarrow E_{7}$ : incomplete case when $p_{1}$ is blown up first and then $p_{2}$ is blown up $\left(p_{0} \rightarrow p_{1} \rightarrow p_{2}\right)$. The singularities appearing at each step of the process are shown with their homogeneous coordinates on $\mathbb{P}^{2}$. The ones marked by a circle are those blown up at the subsequent processes. $p_{0}$ denotes the original singularity on the fiber. The notes in the parentheses (such as $\tilde{x}_{1}=-3 s$ for $q_{1}$ ) imply that they are not generically (i.e. unless $s \neq 0$ ) the points on the $\mathbb{P}^{2}$ arising at the respective step of the blowing-up process.

Let us start from defining $\delta_{p_{0}}$ by $\mathcal{C}_{p_{0}}$ in chart 1 (take chart $1_{z}$ : see (3.6)) as

Chart $1_{z}$

$$
\mathcal{C}_{p_{0}}: z=0, y_{1}=0, \quad \delta_{p_{0}}: z=0, y_{1}=0
$$

Since $\mathcal{C}_{p_{0}}$ does not depend on $s, \delta_{p_{0}}$ has the same form as $\mathcal{C}_{p_{0}}$.

Next, we lift them up in chart $2_{z x}$. Lifting up is done by transforming the coordinates from chart $A$ to chart $A^{\prime}$ : in this case, from chart $1_{z}$ to chart $2_{z x}$. The relation between these charts is $\left(\tilde{x}_{1}, y_{1}, z\right)=\left(\tilde{x}_{1}, \tilde{x}_{1} y_{2}, \tilde{x}_{1} z_{2}\right)$ (see (3.7) and (3.8)). Substituting it into (3.20), we see that $\mathcal{C}_{p_{0}}$ and $\delta_{p_{0}}$ are written as $\tilde{x}_{1} z_{2}=0, \tilde{x}_{1} y_{2}=0$. It is reduced to $z_{2}=0, y_{2}=0$, because $\tilde{x}_{1}\left(=x_{1}-s\right)$ parameterizes $\mathcal{C}_{p_{0}}$ and $\delta_{p_{0}}$, and thus takes a non-zero value. Together with $\mathcal{C}_{p_{1}}$ and $\delta_{p_{1}}$ defined in chart $2_{z x}$ (see (3.8)), we have

$$
\begin{array}{ll}
\text { Chart } 2_{z x} & \mathcal{C}_{p_{1}}: \tilde{x}_{1}=0, y_{2}=0, \quad \delta_{p_{1}}: \tilde{x}_{1}=0, y_{2}=0, \\
& \mathcal{C}_{p_{0}}: z_{2}=0, y_{2}=0, \quad \delta_{p_{0}}: z_{2}=0, y_{2}=0 .
\end{array}
$$

Thus $\mathcal{C}_{p_{0}}\left(\delta_{p_{0}}\right)$ intersects with $\mathcal{C}_{p_{1}}\left(\delta_{p_{1}}\right)$ in this chart:

$$
\mathcal{C}_{p_{0}} \cdot \mathcal{C}_{p_{1}} \neq 0, \quad \delta_{p_{0}} \cdot \delta_{p_{1}} \neq 0
$$

Comparing the locus of the singularities (3.8) with the positions of $\mathcal{C}_{i}(3.21)$, we find that $p_{2}=(0,0,0, s)$ is located at the intersection point of $\mathcal{C}_{p_{0}}$ and $\mathcal{C}_{p_{1}}$, while $q_{1}=(-3 s, 0,0, s)$ and $r_{2}=(0,0,-3 s, s)$ are on $\mathcal{C}_{p_{0}}$ and $\mathcal{C}_{p_{1}}$, respectively. As $s \rightarrow 0, q_{1}$ and $r_{2}$ approach $p_{2}$. At $s=0$, they overlap with $p_{2}$ at the intersection point of $\delta_{p_{0}}$ and $\delta_{p_{1}}$. Similarly, in chart $2_{z z}$, we have

Chart $2 z z$

$$
\begin{array}{ll}
\mathcal{C}_{p_{1}}: z=0, y_{2}=0, & \delta_{p_{1}}: z=0, y_{2}=0, \\
\mathcal{C}_{p_{0}}: \text { Invisible }, & \delta_{p_{0}}: \text { Invisible. }
\end{array}
$$

The reason why $\mathcal{C}_{p_{0}}$ and $\delta_{p_{0}}$ are invisible in chart $2_{z z}$ is as follows. As seen from (3.7) and (3.9), the coordinates of $1_{z}$ and $2_{z z}$ are related by $\left(\tilde{x}_{1}, y_{1}, z\right)=\left(x_{2} z, y_{2} z, z\right)$. Then $\mathcal{C}_{p_{0}}$ and $\delta_{p_{0}}(3.20)$ are given by $\left(x_{2} z, y_{2} z, z\right)=\left(\tilde{x}_{1} \neq 0,0,0\right)$. It yields $x_{2}=\infty$, which means that the lift-ups of $\mathcal{C}_{p_{0}}$ and $\delta_{p_{0}}$ cannot be seen in the finite region of chart $2_{z z}$. The locus 
of the singularities $q_{2}$ and $r_{2}$ can be read from (3.9) and (3.23)., For $s \neq 0$, both of them are on $\mathcal{C}_{p_{1}}$. At $s=0, q_{2}$ stays on $\delta_{p_{1}}$, whereas $r_{2}$ has gone to infinity. The positions of the exceptional curves and the singularities in chart 2 are schematically depicted in the leftmost column of figure 1.

In the same way, lifting up from chart $2_{z x}$ to chart $3_{z x x}$ yields

Chart $3_{z x x}$

$$
\begin{array}{ll}
\mathcal{C}_{p_{2}}: \tilde{x}_{1}=0, y_{3}^{2}=3 s z_{3}, & \delta_{p_{2}}: \tilde{x}_{1}=0, y_{3}=0, \\
\mathcal{C}_{p_{1}}: \text { Invisible }, & \delta_{p_{1}}: \text { Invisible }, \\
\mathcal{C}_{p_{0}}: z_{3}=0, y_{3}=0, & \delta_{p_{0}}: z_{3}=0, y_{3}=0 .
\end{array}
$$

This leads to

$$
\mathcal{C}_{p_{0}} \cdot \mathcal{C}_{p_{2}} \neq 0, \quad \delta_{p_{0}} \cdot \delta_{p_{2}} \neq 0 .
$$

The singularity $q_{1}=(-3 s, 0,0, s)(3.10)$ is contained in $\mathcal{C}_{p_{0}}$ but not in $\mathcal{C}_{p_{2}}$ for $s \neq 0$, whereas it is located at the intersection point of $\delta_{p_{0}}$ and $\delta_{p_{2}}$ at $s=0$. Lift-up from chart $2_{z x}$ to chart $3_{z x z}$ yields

Chart $3_{z x z}$

$$
\begin{array}{ll}
\mathcal{C}_{p_{2}}: z_{2}=0, y_{3}^{2}=3 s \tilde{x}_{3}, & \delta_{p_{2}}: z_{2}=0, y_{3}=0, \\
\mathcal{C}_{p_{1}}: \tilde{x}_{3}=0, y_{3}=0, & \delta_{p_{1}}: \tilde{x}_{3}=0, y_{3}=0, \\
\mathcal{C}_{p_{0}}: \text { Invisible }, & \delta_{p_{0}}: \text { Invisible },
\end{array}
$$

and hence

$$
\mathcal{C}_{p_{1}} \cdot \mathcal{C}_{p_{2}} \neq 0, \quad \delta_{p_{1}} \cdot \delta_{p_{2}} \neq 0 .
$$

The singularity $r_{2}=(0,0,-3 s, s)(3.11)$ is contained in $\mathcal{C}_{p_{1}}$ but not in $\mathcal{C}_{p_{2}}$ for $s \neq 0$, whereas it is located at the intersection point of $\delta_{p_{1}}$ and $\delta_{p_{2}}$ at $s=0$. The positions of these objects in chart 3 (together with the objects in chart $2_{z z}$ ) are depicted in the second column of figure 1 .

As seen in the previous subsection, the remaining three singularities $q_{1}$ in chart $3_{z x x}, r_{2}$ in chart $3_{z x z}$ and $q_{2}$ in chart $2_{z z}$ are independently blown up. Here we consider the blow-up of $q_{1}$ and lift all the information of chart $3_{z x x}$ (3.24) up in chart 4. In chart $4_{z x x x}$ (3.13), the result is

$$
\begin{array}{lll}
\text { Chart } 4_{z x x x} & \mathcal{C}_{q_{1}}: \tilde{\tilde{x}}_{1}=0, y_{4}^{2}=z_{4}-3 z_{4}^{2} s, & \delta_{q_{1}}: \tilde{\tilde{x}}_{1}=0, y_{4}^{2}=z_{4}, \\
& \mathcal{C}_{p_{2}}: \tilde{\tilde{x}}_{1}-3 s=0, y_{4}^{2}=z_{4}, & \delta_{p_{2}}: \text { Invisible } \\
& \mathcal{C}_{p_{1}}: \text { Invisible, } & \delta_{p_{1}}: \text { Invisible }, \\
& \mathcal{C}_{p_{0}}: z_{4}=0, y_{4}=0, & \delta_{p_{0}}: z_{4}=0, y_{4}=0 .
\end{array}
$$

We will briefly explain how the forms of $\mathcal{C}_{p_{2}}$ and $\delta_{p_{2}}$ are obtained. The coordinates of chart $3_{z x x}$ and $4_{z x x x}$ are related by (see (3.12) and (3.13))

$$
\left(\tilde{x}_{1}+3 s, y_{3}, z_{3}\right)=\left(\tilde{\tilde{x}}_{1}, \tilde{\tilde{x}}_{1} y_{4}, \tilde{\tilde{x}}_{1} z_{4}\right) .
$$

Substituting it into (3.24), we have

$$
\mathcal{C}_{p_{2}}: \tilde{\tilde{x}}_{1}-3 s=0, \tilde{\tilde{x}}_{1}^{2} y_{4}^{2}=3 s \tilde{\tilde{x}}_{1} z_{4} .
$$


$\mathrm{s} \neq 0$
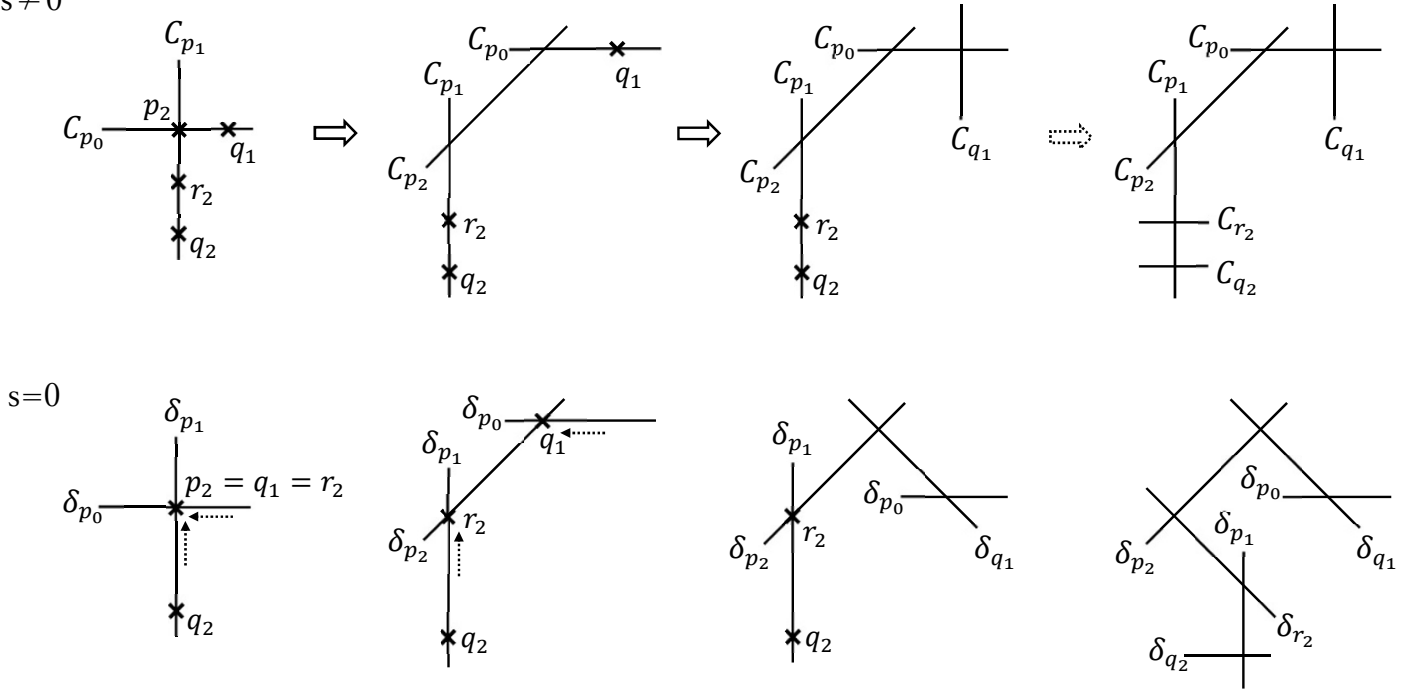

Figure 1. Exceptional curves and singularities of $\mathrm{SO}(12) \rightarrow E_{7}: p_{0} \rightarrow p_{1} \rightarrow p_{2}$ case.

The second equation is rewritten by using the first equation as $\tilde{\tilde{x}}_{1}^{2} y_{4}^{2}=\tilde{\tilde{x}}_{1}^{2} z_{4}$. Since $s \neq 0$, the first equation leads $\tilde{\tilde{x}}_{1} \neq 0$. Thus the second equation is reduced to $y_{4}^{2}=z_{4}$. To see the form of $\delta_{p_{2}}$, we set $s=0$ in (3.29). Then, from (3.24), $\delta_{p_{2}}$ is given by $\left(\tilde{\tilde{x}}_{1}, \tilde{\tilde{x}}_{1} y_{4}, \tilde{\tilde{x}}_{1} z_{4}\right)=$ $\left(0,0, z_{3} \neq 0\right)$. It yields $z_{4}=\infty$ and is invisible in chart $4_{z x x x}$. From (3.28), one can see the following intersections in this chart:

$$
\mathcal{C}_{p_{0}} \cdot \mathcal{C}_{p_{2}} \neq 0, \mathcal{C}_{p_{0}} \cdot \mathcal{C}_{q_{1}} \neq 0, \quad \delta_{p_{0}} \cdot \delta_{q_{1}} \neq 0
$$

In chart $4_{z x x y}$, one can show that $\mathcal{C}_{p_{0}}$ is invisible as well, and no intersection can be seen. In chart $4_{z x x z}(3.14)$, we have

$$
\begin{array}{lll}
\text { Chart } 4_{z x x z} & \mathcal{C}_{q_{1}}: z_{3}=0, y_{4}^{2}=x_{4}-3 s, & \delta_{q_{1}}: z_{3}=0, y_{4}^{2}=x_{4}, \\
& \mathcal{C}_{p_{2}}: x_{4} z_{3}-3 s=0, y_{4}^{2}=x_{4}, & \delta_{p_{2}}: x_{4}=0, y_{4}=0, \\
& \mathcal{C}_{p_{1}}: \text { Invisible }, & \delta_{p_{1}}: \text { Invisible }, \\
\mathcal{C}_{p_{0}}: \text { Invisible }, & \delta_{p_{0}}: \text { Invisible },
\end{array}
$$

where $\left(\tilde{x}_{1}+3 s, y_{3}, z_{3}\right)=\left(x_{4} z_{3}, y_{4} z_{3}, z_{3}\right) . \quad \mathcal{C}_{q_{1}}$ does not intersect with $\mathcal{C}_{p_{2}}$, whereas $\delta_{q_{1}}$ intersects with $\delta_{p_{2}}$ :

$$
\delta_{p_{2}} \cdot \delta_{q_{1}} \neq 0
$$

There is no singularity in these charts. The positions of the exceptional curves and the singularities after this blow-up are given in the third column in figure 1. Since $q_{1}$ was on $\mathcal{C}_{p_{0}}$ but not on $\mathcal{C}_{p_{2}}$ in chart $3, \mathcal{C}_{q_{1}}$ intersects only with $\mathcal{C}_{p_{0}}$ in chart 4 . On the other hand, $q_{1}$ was on the intersection of $\delta_{p_{0}}$ and $\delta_{p_{2}}$, and hence $\delta_{q_{1}}$ bridges them after the blow-up.

The intersections after blowing up the remaining two singularities $r_{2}$ and $q_{2}$ are obtained in a similar manner. The result is given in the rightmost column in figure 1 . The 
final intersection patterns for $s \neq 0$ and $s=0$ are the $D_{6}$ and $A_{6}$ Dynkin diagrams, respectively (see also figure 2 in the next subsection; the meaning of the triangular node in $A_{6}$ will be clarified there).

\subsubsection{Intersection diagram at $s=0$ : transmutation of a root into a weight}

In this subsection, we examine how $D_{6}$ Dynkin diagram at $s \neq 0$ becomes $A_{6}$ at $s=0$; in other words, how the sets $\left\{\mathcal{C}_{I}\right\}$ and $\left\{\delta_{I}\right\}$ are related. For this, as discovered in [11], it is important to know the $s \rightarrow 0$ limit of $\mathcal{C}$, which we write $\lim _{s \rightarrow 0} \mathcal{C}$. Here, we give a detailed explanation how this limit is explicitly calculated. It is worth noting that $\lim _{s \rightarrow 0} \mathcal{C}$ does not necessarily coincide with $\delta$. Suppose $\mathcal{C}$ and $\delta$ are defined in chart $A$, while $\mathcal{C}^{\prime}$ and $\delta^{\prime}$ are defined in a "deeper" chart $A^{\prime}$. By definition, $\lim _{s \rightarrow 0} \mathcal{C}=\delta$ in chart $A$. However, after $\mathcal{C}$ and $\delta$ are lifted up in chart $A^{\prime}, \mathcal{C}$ coexists with $\delta^{\prime}$ and hence $\lim _{s \rightarrow 0} \mathcal{C}$ may contain $\delta^{\prime}$. This can happen only after the lift-up. That is, lifting up and taking $s \rightarrow 0$ limit do not commute in general, and we should take $s \rightarrow 0$ after the lift-up.

Since the lift-ups have been completed in the previous subsection, all that is left is letting $s \rightarrow 0$. Suppose the limit is taken in a chart. As seen in the previous subsection, some of the lift-ups of $\mathcal{C}$ 's and/or $\delta$ 's may be invisible; that is, the limit consists only of the components visible in that chart. Thus the limit should be taken in every chart and the final form of $\lim _{s \rightarrow 0} \mathcal{C}$ is obtained as their union.

In chart 1 and chart 2 , we can see from (3.20), (3.21) and (3.23) that $\lim _{s \rightarrow 0} \mathcal{C}_{i}=\delta_{i}$ for $i=p_{0}, p_{1}$. In chart 3 , we encounter the first nontrivial result. From (3.24), we find in chart $3_{z x x}$ as

$$
\lim _{s \rightarrow 0} \mathcal{C}_{p_{0}}=\delta_{p_{0}}, \quad \lim _{s \rightarrow 0} \mathcal{C}_{p_{2}}=\left\{\tilde{x}_{1}=0, y_{3}^{2}=0\right\}=2 \delta_{p_{2}} .
$$

$\mathcal{C}_{p_{2}}$ has only one component for $s \neq 0$, but after $s \rightarrow 0$, it splits into two overlapping (multiplicity two) $\delta_{p_{2}}$ 's. Similarly, from (3.26), we find in chart $3_{z x z}$ as

$$
\lim _{s \rightarrow 0} \mathcal{C}_{p_{1}}=\delta_{p_{1}}, \quad \lim _{s \rightarrow 0} \mathcal{C}_{p_{2}}=\left\{z_{2}=0, y_{3}^{2}=0\right\}=2 \delta_{p_{2}} .
$$

In chart $3_{z x y}, \lim _{s \rightarrow 0} \mathcal{C}_{p_{2}}$ is invisible.

Next we consider charts $4_{z x x *}$ arising from the blow-up of $q_{1}$. In chart $4_{z x x x}, \mathcal{C}_{p_{2}}$ is visible, but $\delta_{p_{2}}$ is invisible (see (3.28)). Nevertheless, $\lim _{s \rightarrow 0} \mathcal{C}_{p_{2}}$ does exist. Actually, one can see from (3.28) that

$$
\lim _{s \rightarrow 0} \mathcal{C}_{p_{2}}=\delta_{q_{1}} .
$$

Also, we find $\lim _{s \rightarrow 0} \mathcal{C}_{p_{0}}=\delta_{p_{0}}, \lim _{s \rightarrow 0} \mathcal{C}_{q_{1}}=\delta_{q_{1}}$. One can show that the same results are obtained in chart $4_{z x x y}$ except that $\mathcal{C}_{p_{0}}$ is invisible. In chart $4_{z x x z}$, one can see from (3.32) that $\mathcal{C}_{p_{2}}$ splits into two components as follows:

$$
\begin{aligned}
\lim _{s \rightarrow 0} \mathcal{C}_{p_{2}} & =\left\{x_{4} z_{3}=0, y_{4}^{2}=x_{4}\right\} \\
& =\left\{x_{4}=0, y_{4}^{2}=0\right\} \cup\left\{z_{3}=0, y_{4}^{2}=x_{4}\right\} \\
& =2 \delta_{p_{2}}+\delta_{q_{1}} .
\end{aligned}
$$

Also, $\lim _{s \rightarrow 0} \mathcal{C}_{q_{1}}=\delta_{q_{1}}$ is satisfied. 
In charts $4_{z x z *}$, which arise from the blow-up of $r_{2}$, one can show by repeating the same argument that

$$
\begin{array}{ll}
\lim _{s \rightarrow 0} \mathcal{C}_{p_{2}}=2 \delta_{p_{2}}+\delta_{r_{2}} & \left(\operatorname{chart} 4_{z x z x}\right), \\
\lim _{s \rightarrow 0} \mathcal{C}_{p_{2}}=\delta_{r_{2}}, & \left(\operatorname{chart} 4_{z x z y}, 4_{z x z z}\right) .
\end{array}
$$

All the other curves satisfy $\lim _{s \rightarrow 0} \mathcal{C}_{i}=\delta_{i}$.

Finally, after blowing up $q_{2}$, charts $3_{z z *}$ contain $\mathcal{C}_{q_{2}}$ as well as the lift-ups of $\mathcal{C}_{p_{0}}$ and $\mathcal{C}_{p_{1}}$. A similar analysis shows that $\lim _{s \rightarrow 0} \mathcal{C}_{i}=\delta_{i}$ for $i=p_{0}, p_{1}$ and $q_{2}$.

Collecting all the above results, we find only $\mathcal{C}_{p_{2}}$ has a non-trivial limit. The final form of $\lim _{s \rightarrow 0} \mathcal{C}_{p_{2}}$ is given by the union of the components that are visible in each chart, and hence from (3.34), (3.35), (3.36), (3.37) and (3.38),

$$
\lim _{s \rightarrow 0} \mathcal{C}_{p_{2}}=2 \delta_{p_{2}}+\delta_{q_{1}}+\delta_{r_{2}} .
$$

In conclusion, we find (Hereafter, $\lim _{s \rightarrow 0}$ will be omitted.)

$$
\begin{aligned}
& \mathcal{C}_{p_{0}}=\delta_{p_{0}}, \\
& \mathcal{C}_{p_{1}}=\delta_{p_{1}}, \\
& \mathcal{C}_{p_{2}}=2 \delta_{p_{2}}+\delta_{q_{1}}+\delta_{r_{2}}, \\
& \mathcal{C}_{q_{1}}=\delta_{q_{1}} \\
& \mathcal{C}_{r_{2}}=\delta_{r_{2}}, \\
& \mathcal{C}_{q_{2}}=\delta_{q_{2}}
\end{aligned}
$$

The intersection matrix of $\mathcal{C}$ 's is the minus of the $D_{6}=\mathrm{SO}(12)$ Cartan matrix:

$$
-\mathcal{C}_{I} \cdot \mathcal{C}_{J}=\left(\begin{array}{cccccc}
2 & -1 & 0 & 0 & 0 & 0 \\
-1 & 2 & -1 & 0 & 0 & 0 \\
0 & -1 & 2 & -1 & 0 & 0 \\
0 & 0 & -1 & 2 & -1 & -1 \\
0 & 0 & 0 & -1 & 2 & 0 \\
0 & 0 & 0 & -1 & 0 & 2
\end{array}\right)
$$

where $I, J=q_{1}, p_{0}, p_{2}, p_{1}, r_{2}, q_{2}$ in this order. Note that this is different from the order of the blow-ups (see the upper rightmost diagram in figure 1, or equivalently, the upper diagram in figure 2 below). From (3.40), $\delta$ 's are expressed in terms of $\mathcal{C}$ 's. Then one can compute the intersection matrix of $\delta$ 's, which is found to be

$$
-\delta_{I} \cdot \delta_{J}=\left(\begin{array}{cccccc}
2 & -1 & -1 & 0 & 0 & 0 \\
-1 & 2 & 0 & 0 & 0 & 0 \\
-1 & 0 & \frac{3}{2} & 0 & -1 & 0 \\
0 & 0 & 0 & 2 & -1 & -1 \\
0 & 0 & -1 & -1 & 2 & 0 \\
0 & 0 & 0 & -1 & 0 & 2
\end{array}\right) .
$$



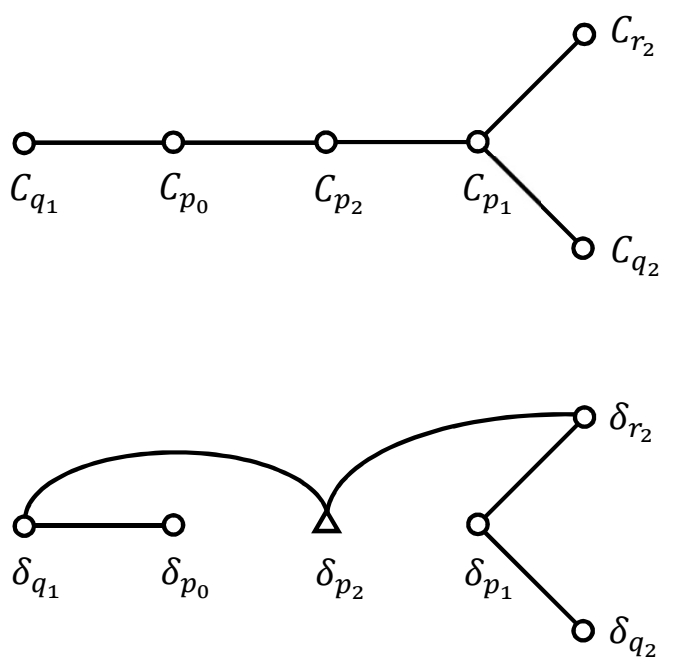

Figure 2. Generic $\mathrm{SO}(12)$ intersection diagram at $s \neq 0$ (upper) and incomplete intersection diagram at $s=0$ (lower) of $\mathrm{SO}(12) \rightarrow E_{7}: p_{1}$-first $\left(p_{0} \rightarrow p_{1} \rightarrow p_{2}\right)$ case.

This is the minus of the $A_{6}=\mathrm{SU}(7)$ Cartan matrix, except that the self-intersection number of $\delta_{p_{2}}$ is not -2 , but $-\frac{3}{2}$. Namely, it is true that the intersection pattern of $\delta$ 's is given by the $A_{6}$ Dynkin diagram, but their intersection "numbers" are slightly different from the corresponding $A_{6}$ Cartan matrix. This difference is expressed in the lower diagram in figure 2 as the triangular (not circular) node. In summary,

$$
\begin{aligned}
& \text { intersection pattern } \Rightarrow A_{6} \text { Dynkin diagram, } \\
& \text { intersection numbers } \Rightarrow A_{6} \text { Dynkin diagram with a triangular node. }
\end{aligned}
$$

Let us call the latter diagram "intersection diagram". Since it contains a $-\frac{3}{2}$ node, it is not a usual $A_{6}$ Dynkin diagram. In this sense, we conclude in the present case that the intersection diagram is an $A_{6}$ "non-Dynkin" diagram.

In terms of $\mathcal{C}$ 's, $\delta_{p_{2}}$ is written as $\delta_{p_{2}}=\frac{1}{2}\left(\mathcal{C}_{p_{2}}-\mathcal{C}_{q_{1}}-\mathcal{C}_{r_{2}}\right)$. This is one of the weights in the spinor representation of $\mathrm{SO}(12)$. Thus we see that at a generic $s \neq 0$ codimension-one locus of the singularity the exceptional fibers after the resolutions form a root system of $\mathrm{SO}(12)$, but at $s=0$ one of the simple roots $\left(\mathcal{C}_{p_{2}}\right)$ is transmuted to a weight in the spinor representation $\left(\delta_{p_{2}}\right)$.

These $\delta$ 's form a basis of the two-cycles appearing at the codimension-two singularity after the resolution. Let us consider the lattice spanned by $\delta$ 's:

$$
J=\sum_{I=q_{1}, p_{0}, p_{2}, p_{1}, r_{2}, q_{2}} n_{I} \delta_{I} \quad\left(n_{I} \in Z\right),
$$

where each lattice point expresses a two-cycle at $s=0$. One can show by using (3.42) that

$$
\begin{aligned}
& \sharp(J \cdot J=-2)=60, \\
& \sharp\left(J \cdot J=-\frac{3}{2}\right)=32 .
\end{aligned}
$$




\begin{tabular}{|c|c|c|c|c|}
\hline & 1st blow up & 2nd blow up & 3rd blow up & 4th blow up \\
\hline$p_{0} \rightarrow$ & $\begin{array}{l}p_{1}\left(s^{2}: 0: 1\right) \rightarrow \\
q_{1}\left(-2 s^{2}: 0: 1\right)\end{array}$ & $\begin{array}{l}p_{2}(1: 0: 0)\left(\text { in } 2_{z x}\right) \rightarrow \\
q_{1}(1: 0: 0)\left(\tilde{x}_{1}=-3 s^{2}\right) \\
r_{2}\left(1: 0:-3 s^{2}\right) \\
q_{2}(0: 0: 1)(\text { in } 2 z z) \rightarrow\end{array}$ & $\begin{array}{l}p_{3}(1: 0: 0 ; s=0)(\operatorname{codim} .2) \rightarrow \\
q_{1}(1: 0: 0)\left(\tilde{x}_{1}=-3 s^{2}\right) \rightarrow \\
r_{2}(0: 0: 1)\left(z_{2}=-3 s^{2}\right) \rightarrow \\
\text { regular }\end{array}$ & $\begin{array}{l}\text { regular } \\
\text { regular } \\
\text { regular }\end{array}$ \\
\hline
\end{tabular}

Table 2. $\mathrm{SO}(12) \rightarrow E_{7}$ : complete case when $p_{1}$ is blown up first $\left(p_{0} \rightarrow p_{1} \rightarrow p_{2}\right)$. The new isolated codimension-two conifold singularity is shown in red.

They respectively correspond to the adjoint representation (except the Cartan part) of $\mathrm{SO}(12)$ and the spinor representation 32 of $\mathrm{SO}(12)$. The latter representation consists of 16 states with $n_{I} \geq 0$ for all $I$ and 16 states with $n_{I} \leq 0$ for all $I$. Note that, unlike in the cases of the ordinary (the complete) resolutions (1.1), there appears only one irreducible representation $(=\mathbf{3 2})$ in the integer span of the two-cycles at the singularity, indicating that it is a half-hypermultiplet.

\subsection{Complete resolution: blowing up $p_{1}$ first}

\subsubsection{Blowing up process}

The geometry of complete resolution is given by setting $H_{n-r+4}=s^{2}$ instead of $H_{n-r+4}=s$ in (2.13) and (2.14) (the other polynomials are unchanged), we have

$$
\Phi(x, y, z, s)=-y^{2}+x^{3}+f(z, s) x+g(z, s)=0,
$$

where

$$
\begin{aligned}
& f(z, s)=-3 s^{4} z^{2}+z^{3} \\
& g(z, s)=2 s^{6} z^{3}-s^{2} z^{4} .
\end{aligned}
$$

Singularities can be blown up in the same way as in section 3.1.1. As a result, a new isolated (codimension-two) singularity $p_{3}$ arises in chart 3 after $p_{2}$ is blown up in chart $2_{z x}$. Regarding the structure of singularities, this is the only difference between complete and incomplete cases (see table 2 below).

Explicitly, the 3rd blow up (3.10) and (3.11) are replaced as follows:

\section{3rd blow up.}

Chart $3_{z x x}$

$$
\begin{aligned}
\Psi_{z x}\left(\tilde{x}_{1}, \tilde{x}_{1} y_{3}, \tilde{x}_{1} z_{3}, s\right) & =\tilde{x}_{1}^{2} \Psi_{z x x}\left(\tilde{x}_{1}, y_{3}, z_{3}, s\right) \\
\Psi_{z x x}\left(\tilde{x}_{1}, y_{3}, z_{3}, s\right) & =z_{3}\left(3 s^{2}+\tilde{x}_{1} z_{3}+\tilde{x}_{1}\right)-y_{3}^{2} . \\
\mathcal{C}_{p_{2}} \text { in } 3_{z x x} & : \tilde{x}_{1}=0, y_{3}^{2}=3 s^{2} z_{3} . \\
\text { Singularities } & :\left(\tilde{x}_{1}, y_{3}, z_{3}, s\right)=\left(-3 s^{2}, 0,0, s\right)\left(=q_{1}\right),(0,0,-1,0)\left(=p_{3}\right) .
\end{aligned}
$$

Chart $3_{z x y}$ Regular. 
Chart $3_{z x z}$

$$
\begin{aligned}
\Psi_{z x}\left(x_{3} z_{2}, y_{3} z_{2}, z_{2}, s\right) & =z_{2}^{2} \Psi_{z x z}\left(x_{3}, y_{3}, z_{2}, s\right) \\
\Psi_{z x z}\left(x_{3}, y_{3}, z_{2}, s\right) & =3 s^{2} x_{3}+x_{3}\left(x_{3}+1\right) z_{2}-y_{3}^{2} . \\
\mathcal{C}_{p_{2}} \text { in } 3_{z x z} & : z_{2}=0, y_{3}^{2}=3 s^{2} x_{3} . \\
\text { Singularities } & :\left(x_{3}, y_{3}, z_{2}, s\right)=\left(0,0,-3 s^{2}, s\right)\left(=r_{2}\right),(-1,0,0,0)\left(=p_{3}\right) .
\end{aligned}
$$

The new isolated singularity $p_{3}$ is the conifold singularity. To see this, consider chart $3_{z x x}(3.48)$ and shift $p_{3}$ to the origin via

$$
\tilde{z}_{3} \equiv z_{3}+1
$$

The defining equation then reads

$$
\Upsilon_{z x x}\left(\tilde{x}_{1}, y_{3}, \tilde{z}_{3}, s\right) \equiv \Psi_{z x x}\left(\tilde{x}_{1}, y_{3}, \tilde{z}_{3}-1, s\right) .
$$

The explicit form is

$$
\Upsilon_{z x x}\left(\tilde{x}_{1}, y_{3}, \tilde{z}_{3}, s\right)=-y_{3}^{2}+\left(-1+\tilde{z}_{3}\right)\left(\tilde{x}_{1} \tilde{z}_{3}+3 s^{2}\right),
$$

where $p_{3}$ is located at $\left(\tilde{x}_{1}, y_{3}, \tilde{z}_{3}, s\right)=(0,0,0,0)$. The leading terms of $\Upsilon_{z x x}$ can be written as $\Upsilon_{z x x} \sim \tilde{z}_{3}\left(-\tilde{x}_{1}\right)-\left(y_{3}+i \sqrt{3} s\right)\left(y_{3}-i \sqrt{3} s\right) \sim X_{1} X_{4}-X_{2} X_{3}$, and hence $\Upsilon_{z x x}=0$ is a conifold. Including the subleading terms, we have

$$
\begin{aligned}
\Upsilon_{z x x}\left(\tilde{x}_{1}, y_{3}, \tilde{z}_{3}, s\right) & =\tilde{z}_{3}\left(-\tilde{x}_{1}+\tilde{x}_{1} \tilde{z}_{3}+3 s^{2}\right)-\left(y_{3}+i \sqrt{3} s\right)\left(y_{3}-i \sqrt{3} s\right) \\
& =X_{1} X_{4}-X_{2} X_{3},
\end{aligned}
$$

where $X_{i}$ are given by ${ }^{5}$

$$
\begin{aligned}
& X_{1}=\tilde{z}_{3}, \\
& X_{4}=-\tilde{x}_{1}+\tilde{x}_{1} \tilde{z}_{3}+3 s^{2}, \\
& X_{2}=y_{3}+i \sqrt{3} s, \\
& X_{3}=y_{3}-i \sqrt{3} s .
\end{aligned}
$$

$p_{3}$ is located at the origin $\left(X_{1}, X_{2}, X_{3}, X_{4}\right)=(0,0,0,0)$, which is the conifold singularity.

The exceptional curves existing in chart $3_{z x x}$ are given by replacing $s$ to $s^{2}$ in (3.24). After the coordinate changes (3.50) and (3.54), their positions read

Chart $3_{z x x}$

$$
\begin{array}{ll}
\mathcal{C}_{p_{2}}: X_{4}=-3 s^{2}, \frac{\left(X_{2}+X_{3}\right)^{2}}{4}=3 s^{2}\left(X_{1}-1\right), & \delta_{p_{2}}: X_{4}=0, X_{2}=X_{3}=0 \\
\mathcal{C}_{p_{1}}: \text { Invisible }, & \delta_{p_{1}}: \text { Invisible } \\
\mathcal{C}_{p_{0}}: X_{1}=1, X_{2}+X_{3}=0, & \delta_{p_{0}}: X_{1}=1, X_{2}=X_{3}=0
\end{array}
$$

where $s=-\frac{i}{2 \sqrt{3}}\left(X_{2}-X_{3}\right)$. The conifold singularity $p_{3}$ is contained in $\delta_{p_{2}}$, but not in $\delta_{p_{0}}$. Similarly, in chart $3_{z x z}, p_{3}$ is on $\delta_{p_{2}}$, but not on $\delta_{p_{1}}$. As for figure 1 , one new point $p_{3}$ is added on $\delta_{p_{2}}$ in the second figure of the bottom line.

\footnotetext{
${ }^{5}$ One may exchange the definitions of $X_{1}$ and $X_{4}$, or $X_{2}$ and $X_{3}$, but it does not change the result $((3.65)$ below). It also holds for all the other cases discussed in this paper.
} 


\subsubsection{Intersection diagram at $s=0$}

Since $p_{3}$ is a codimension-two singularity, it does not change the intersection diagram for generic $s \neq 0$ (ordinary $\mathrm{SO}(12)$ Dynkin diagram in figure 2). Here we examine how the intersection diagram at $s=0$ is modified via the resolution of $p_{3}$.

The conifold singularity $p_{3}$ is resolved by inserting an exceptional curve $\mathbb{P}^{1}$ at the origin. This process is called the small resolution. We write the inserted $\mathbb{P}^{1}$ as $\delta_{\text {complete }}$.

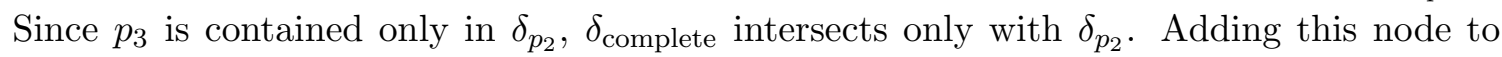
figure 2 , we find that the intersection pattern becomes $E_{7}$ (see figure 3 below). At this stage, however, it is not clear what intersection matrix is associated with this diagram. To clarify it, we need a lift-up.

The smooth geometry after the small resolution is covered by two local coordinate patches $H_{+}$and $H_{-}$(see appendix C). By lifting up the relevant objects from chart 3 into these patches, one can examine how the limit $s \rightarrow 0$ is modified from the incomplete case and what intersection matrix is obtained. Since $p_{3}$ is separated from $\delta_{p_{0}}$ and $\delta_{p_{1}}$, lifting up $\mathcal{C}_{p_{2}}$ and $\delta_{p_{2}}$ is sufficient. We consider chart $3_{z x x}(3.55)$.

The local coordinates of $H_{+}$are $\left(X_{2}^{\prime}, X_{4}^{\prime}, \lambda\right)$ and the resolved geometry is obtained by the replacement (C.4):

$$
\left(X_{1}, X_{2}, X_{3}, X_{4}\right)=\left(-\lambda X_{2}^{\prime}, X_{2}^{\prime},-\lambda X_{4}^{\prime}, X_{4}^{\prime}\right)
$$

Here we put ' for the coordinates after the small resolution. The explicit form of $\delta_{\text {complete }}$ in chart $H_{+}$is given by (C.5). The lift-ups of $\mathcal{C}_{p_{2}}$ and $\delta_{p_{2}}$ are given by substituting (3.56) into (3.55). Then we have

Chart $H_{+}$

$$
\begin{array}{ll}
\mathcal{C}_{p_{2}}: X_{4}^{\prime}=-3 s^{2}, \frac{\left(X_{2}^{\prime}-\lambda X_{4}^{\prime}\right)^{2}}{4}=3 s^{2}\left(-\lambda X_{2}^{\prime}-1\right), \quad \delta_{p_{2}}: \text { Invisible },
\end{array}
$$

where

$$
s=-\frac{i}{2 \sqrt{3}}\left(X_{2}^{\prime}+\lambda X_{4}^{\prime}\right) .
$$

$\delta_{p_{2}}$ is invisible, since $\delta_{p_{2}}(3.55)$ is given by $\left(-\lambda X_{2}^{\prime}, X_{2}^{\prime},-\lambda X_{4}^{\prime}, X_{4}^{\prime}\right)=\left(X_{1}, 0,0,0\right)$ with $X_{1} \neq 0$, which is impossible. In this patch, $\delta_{p_{2}}$ and $\delta_{\text {complete }}$ do not intersect. The $s \rightarrow 0$ limit is given by the replacement $X_{2}^{\prime}=-\lambda X_{4}^{\prime}$, and hence

$$
\begin{aligned}
\lim _{s \rightarrow 0} \mathcal{C}_{p_{2}} & =\left\{X_{4}^{\prime}=0, \lambda^{2} X_{4}^{\prime 2}=0\right\} \quad \text { with } X_{2}^{\prime}=-\lambda X_{4}^{\prime} \\
& =\left\{\left(X_{2}^{\prime}, X_{4}^{\prime}, \lambda\right)=(0,0, \lambda)\right\} \\
& =\delta_{\text {complete }}
\end{aligned}
$$

In the other patch $H_{-}$, the local coordinates are $\left(X_{1}^{\prime}, X_{3}^{\prime}, \mu\right)$ and the resolution is given by (C.7):

$$
\left(X_{1}, X_{2}, X_{3}, X_{4}\right)=\left(X_{1}^{\prime},-\mu X_{1}^{\prime}, X_{3}^{\prime},-\mu X_{3}^{\prime}\right)
$$


In this patch, $\delta_{\text {complete }}(\mathrm{C} .8)$ as well as $\mathcal{C}_{p_{2}}$ and $\delta_{p_{2}}$ (3.55) take the following forms:

\section{Chart $H_{-}$}

$$
\begin{array}{ll} 
& \delta_{\text {complete }}:\left(X_{1}^{\prime}, X_{3}^{\prime}, \mu\right)=(0,0, \mu), \\
\mathcal{C}_{p_{2}}: \mu X_{3}^{\prime}=3 s^{2}, \frac{\left(X_{3}^{\prime}-\mu X_{1}^{\prime}\right)^{2}}{4}=3 s^{2}\left(X_{1}^{\prime}-1\right), \quad \delta_{p_{2}}:\left(X_{1}^{\prime}, X_{3}^{\prime}, \mu\right)=\left(X_{1}^{\prime}, 0,0\right),
\end{array}
$$

where

$$
s=\frac{i}{2 \sqrt{3}}\left(X_{3}^{\prime}+\mu X_{1}^{\prime}\right)
$$

This time, $\delta_{p_{2}}$ is visible. $\delta_{p_{2}}$ and $\delta_{\text {complete }}$ are intersecting at $\left(X_{1}^{\prime}, X_{3}^{\prime}, \mu\right)=(0,0,0)$ :

$$
\delta_{p_{2}} \cdot \delta_{\text {complete }} \neq 0
$$

The $s \rightarrow 0$ limit is given by the replacement $X_{3}^{\prime}=-\mu X_{1}^{\prime}$ :

$$
\begin{aligned}
\lim _{s \rightarrow 0} \mathcal{C}_{p_{2}} & =\left\{-\mu^{2} X_{1}^{\prime}=0, \mu^{2} X_{1}^{\prime 2}=0\right\} \quad \text { with } X_{3}^{\prime}=-\mu X_{1}^{\prime} \\
& =\left\{X_{1}^{\prime}=0\right\} \cup\left\{\mu^{2}=0\right\} \quad \text { with } X_{3}^{\prime}=-\mu X_{1}^{\prime} \\
& =\left\{\left(X_{1}^{\prime}, X_{3}^{\prime}, \mu\right)=(0,0, \mu)\right\} \cup\left\{\left(X_{1}^{\prime}, X_{3}^{\prime}, \mu\right)=\left(X_{1}^{\prime}, 0,0\right)\right\}^{\otimes 2} \\
& =\delta_{\text {complete }}+2 \delta_{p_{2}} .
\end{aligned}
$$

In conclusion, for the complete resolution, the new exceptional curve $\delta_{\text {complete }}$ is contained in $\lim _{s \rightarrow 0} \mathcal{C}_{p_{2}}$ with multiplicity one, and (3.40) is modified as

$$
\begin{aligned}
& \mathcal{C}_{p_{0}}=\delta_{p_{0}} \\
& \mathcal{C}_{p_{1}}=\delta_{p_{1}} \\
& \mathcal{C}_{p_{2}}=2 \delta_{p_{2}}+\delta_{q_{1}}+\delta_{r_{2}}+\delta_{\text {complete }} \\
& \mathcal{C}_{q_{1}}=\delta_{q_{1}} \\
& \mathcal{C}_{r_{2}}=\delta_{r_{2}} \\
& \mathcal{C}_{q_{2}}=\delta_{q_{2}}
\end{aligned}
$$

Then, assuming that the intersection matrix of these seven $\delta$ 's is just the minus of ordinary $E_{7}$ Cartan matrix, we find that the intersection matrix of the six $\mathcal{C}$ 's computed by (3.65) is precisely the minus of the $\mathrm{SO}(12)$ Cartan matrix. It means that, in contrast to the incomplete case, $\delta$ 's have no node with self-intersection number $-\frac{3}{2}$ and the intersection diagram is the ordinary $E_{7}$ Dynkin diagram. That is, $\delta_{p_{2}}$, whose self-intersection number is $-\frac{3}{2}$ (triangular node) in the incomplete case, becomes the ordinary node with self-intersection -2 (circular node) by virtue of the existence of $\delta_{\text {complete }}$. The result is summarized in figure 3. As usual, two cycles $J$ spanned by the seven $\delta$ 's with $J \cdot J=-2$ contain two 32's as in (1.1). 


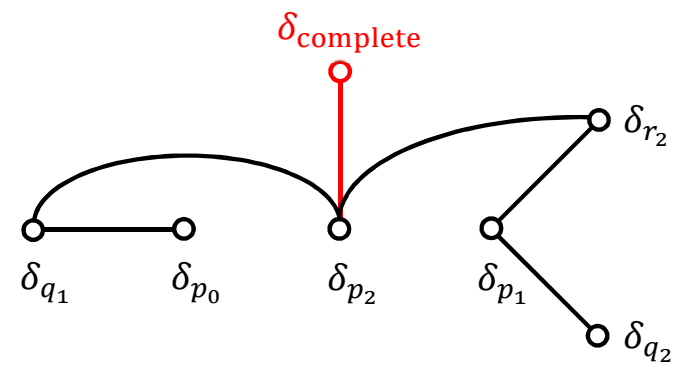

Figure 3. Complete $E_{7}$ intersection diagram of $\mathrm{SO}(12) \rightarrow E_{7}: p_{1}$-first $\left(p_{0} \rightarrow p_{1} \rightarrow p_{2}\right)$ case.

\subsection{Incomplete resolution: blowing up $q_{1}$ first}

\subsubsection{Blowing up process}

In section 3.1, between the two singularities $p_{1}$ and $q_{1}$ arising from the first blow up in chart $1_{z}(3.6), p_{1}$ was blown up first. In this section, let us blow up $q_{1}$ first and see the differences. This time we make a shift of the coordinate $x_{1}$ so that $q_{1}$ comes to $(0,0,0, s)$ : we define

$$
\Sigma_{z}\left(\tilde{x}_{1}, y_{1}, z, s\right) \equiv \Phi_{z}\left(\tilde{x}_{1}-2 s, y_{1}, z, s\right)
$$

$\Sigma_{z}\left(\tilde{x}_{1}, y_{1}, z, s\right)=0$ has singularities $(3 s, 0,0, s)\left(=p_{1}\right)$ and $(0,0,0, s)\left(=q_{1}\right)$. We blow up the latter singularity. The process is completely parallel to that in the previous section so we will only describe the relevant charts and show the main differences from the previous case. For later use, we also present the definition of $\delta$ and the lift-ups of $\mathcal{C}$ and $\delta$. For the $p_{1}$-first case studied in the previous sections, the exceptional curves arising from the blow-ups of the singularities $p_{0}, p_{1}, p_{2}, q_{1}, r_{2}$ and $q_{2}$ are respectively expressed by $\mathcal{C}_{p_{0}}, \mathcal{C}_{p_{1}}$, $\mathcal{C}_{p_{2}}, \mathcal{C}_{q_{1}}, \mathcal{C}_{r_{2}}$ and $\mathcal{C}_{q_{2}}$. We use the same notation for the $q_{1}$-first case as well.

2nd blow up. From the blow-up of $q_{1}, \mathcal{C}_{q_{1}}$ arises. $\mathcal{C}_{p_{0}}$ and $\delta_{p_{0}}$ are lifted up from chart $1_{z}$.

Chart $2_{z x}$

$$
\begin{array}{rlrl}
\Sigma_{z}\left(\tilde{x}_{1}, \tilde{x}_{1} y_{2}, \tilde{x}_{1} z_{2}, s\right) & =\tilde{x}_{1}^{2} \Sigma_{z x}\left(\tilde{x}_{1}, y_{2}, z_{2}, s\right) \\
\Sigma_{z x}\left(\tilde{x}_{1}, y_{2}, z_{2}, s\right) & =z_{2}\left(3 s-\tilde{x}_{1}\right)\left(3 s-\tilde{x}_{1}-z_{2}\right)-y_{2}^{2} . & \\
\mathcal{C}_{q_{1}} & : \tilde{x}_{1}=0, \quad 3 s z_{2}\left(3 s-z_{2}\right)-y_{2}^{2}=0, & \delta_{q_{1}}: \tilde{x}_{1}=0, y_{2}=0 \\
\mathcal{C}_{p_{0}} & : z_{2}=0, \quad y_{2}=0, & \delta_{p_{0}}: z_{2}=0, y_{2}=0 . \\
\text { Singularities } & :\left(\tilde{x}_{1}, y_{2}, z_{2}, s\right)=(3 s, 0,0, s)\left(=p_{1}\right) . &
\end{array}
$$

The positions of these objects are depicted in the leftmost column of figure 4 . There are no other singularities in chart $2_{z y}$ or $2_{z z}$, so we blow up $p_{1}$ in chart $2_{z x}$. Again, we need to shift the coordinate so that the singularity we now blow up comes to the origin:

$$
\Psi_{z x}\left(\tilde{\tilde{x}}_{1}, y_{2}, z_{2}, s\right) \equiv \Sigma_{z x}\left(\tilde{\tilde{x}}_{1}+3 s, y_{2}, z_{2}, s\right)
$$




\begin{tabular}{|c|c|c|c|c|}
\hline & 1st blow up & 2nd blow up & 3rd blow up & 4th blow up \\
\hline$p_{0} \rightarrow$ & $\begin{array}{l}q_{1}(-2 s: 0: 1) \rightarrow \\
p_{1}(s: 0: 1)\end{array}$ & $\begin{array}{l}\text { regular } \\
p_{1}(1: 0: 0)\left(\tilde{x}_{1}=3 s\right) \rightarrow\end{array}$ & $\begin{array}{l}p_{2}(1: 0: 0) \rightarrow \\
q_{2}(0: 0: 1) \rightarrow \\
r_{2}(1: 0:-1) \rightarrow\end{array}$ & $\begin{array}{l}\text { regular } \\
\text { regular } \\
\text { regular }\end{array}$ \\
\hline
\end{tabular}

Table 3. $\mathrm{SO}(12) \rightarrow E_{7}$ : incomplete case when $q_{1}$ is blown up first $\left(p_{0} \rightarrow q_{1}\right)$.

3rd blow up. The relevant charts are $3_{z x x}$ and $3_{z x z}$. From the blow-up of $p_{1}, \mathcal{C}_{p_{1}}$ arises.

Chart $3_{z x x}$

$$
\begin{array}{rlrl}
\Psi_{z x}\left(\tilde{\tilde{x}}_{1}, \tilde{\tilde{x}}_{1} y_{3}, \tilde{\tilde{x}}_{1} z_{3}, s\right) & =\tilde{\tilde{x}}_{1}^{2} \Psi_{z x x}\left(\tilde{\tilde{x}}_{1}, y_{3}, z_{3}, s\right) \\
\Psi_{z x x}\left(\tilde{\tilde{x}}_{1}, y_{3}, z_{3}, s\right) & =\tilde{\tilde{x}}_{1} z_{3}\left(z_{3}+1\right)-y_{3}^{2} . & \\
\mathcal{C}_{p_{1}} & : \tilde{\tilde{x}}_{1}=0, \quad y_{3}=0, \quad \tilde{\tilde{x}}_{1}=0, y_{3}=0 \\
\mathcal{C}_{q_{1}} & : \tilde{\tilde{x}}_{1}=-3 s, \quad \tilde{\tilde{x}}_{1} z_{3}\left(1+z_{3}\right)-y_{3}^{2}=0, \quad \delta_{q_{1}}: \text { Invisible, } \\
\mathcal{C}_{p_{0}} & : z_{3}=0, \quad y_{3}=0, & \delta_{p_{0}}: z_{3}=0, y_{3}=0 . \\
\text { Singularities } & :\left(\tilde{\tilde{x}}_{1}, y_{3}, z_{3}, s\right)=(0,0,-1, s)\left(=r_{2}\right),(0,0,0, s)\left(=p_{2}\right) .
\end{array}
$$

Chart $3_{z x z}$

$$
\begin{array}{rlrl}
\Psi_{z x}\left(x_{3} z_{2}, y_{3} z_{2}, z_{2}, s\right) & =z_{2}^{2} \Psi_{z x z}\left(x_{3}, y_{3}, z_{2}, s\right) & \\
\Psi_{z x x}\left(x_{3}, y_{3}, z_{2}, s\right) & =x_{3}\left(x_{3}+1\right) z_{2}-y_{3}^{2} . & & \\
\mathcal{C}_{p_{1}} & : z_{2}=0, \quad y_{3}=0, & \delta_{p_{1}}: z_{2}=0, y_{3}=0 \\
\mathcal{C}_{q_{1}} & : x_{3} z_{2}=-3 s, \quad x_{3} z_{2}\left(x_{3}+1\right)-y_{3}^{2}=0, & \delta_{q_{1}}: x_{3}=0, y_{3}=0 \\
\mathcal{C}_{p_{0}} & : \text { Invisible, } & \delta_{p_{0}} & : \text { Invisible. }
\end{array}
$$$$
\text { Singularities : }\left(x_{3}, y_{3}, z_{2}, s\right)=(-1,0,0, s)\left(=r_{2}\right),(0,0,0, s)\left(=q_{2}\right) \text {. }
$$

Note that $\mathcal{C}_{q_{1}}$ and $\delta_{q_{1}}$ are independently lifted up from (3.67). The positions of these objects are depicted in the middle column of figure 4. One can see from (3.69) and (3.70) that the remaining three singularities $p_{2}, r_{2}$ and $q_{2}$ are separated for all $s$ (not only for $s \neq 0$ but also for $s=0$ ), and hence they are independently blown up. The whole blowing up process is summarized in table 3 . The resulting intersection patterns are depicted in the rightmost column of figure 4 . For $s \neq 0$, the intersection pattern is the $D_{6}$ Dynkin diagram, which is identical to the one in the $p_{1}$-first case (see the upper rightmost diagram in figure 1 , or the upper diagram in figure 2). The orders of the nodes are the same as well. On the other hand, the intersection pattern at $s=0$ is the $E_{6}$ Dynkin diagram, which is different from the one $\left(A_{6}\right)$ in the $p_{1}$-first case. 
$\mathrm{s} \neq 0$

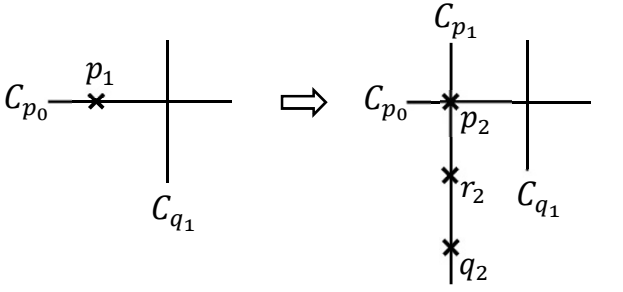

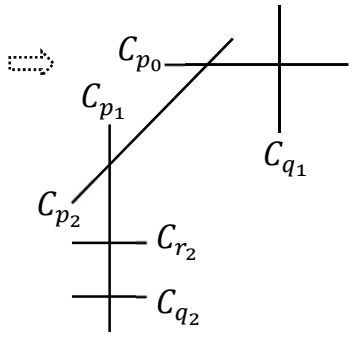

$\mathrm{S}=0$
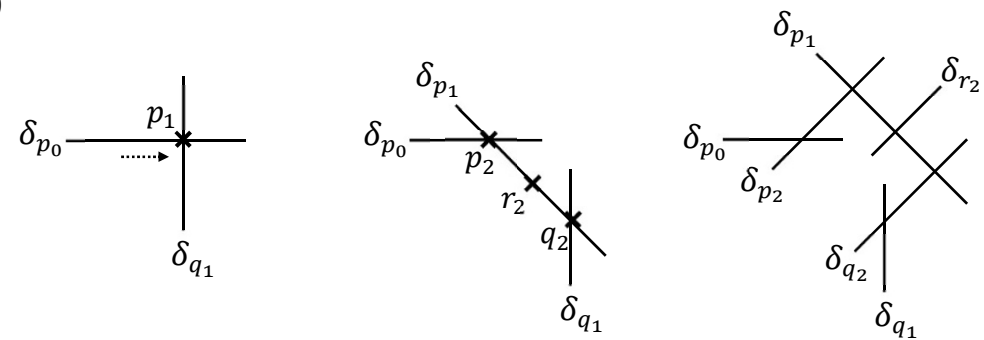

Figure 4. Exceptional curves and singularities of $\mathrm{SO}(12) \rightarrow E_{7}: p_{0} \rightarrow q_{1}$ case.

\subsubsection{Intersection diagram at $s=0$ : differences from the $p_{1}$-first case}

Following the same procedure given in section 3.1.3, we find the $s \rightarrow 0$ limit of $\mathcal{C}$ for the $q_{1}$-first case as

$$
\begin{aligned}
& \mathcal{C}_{p_{0}}=\delta_{p_{0}}, \\
& \mathcal{C}_{q_{1}}=2 \delta_{q_{1}}+2 \delta_{p_{1}}+\delta_{p_{2}}+2 \delta_{q_{2}}+\delta_{r_{2}}, \\
& \mathcal{C}_{p_{1}}=\delta_{p_{1}}, \\
& \mathcal{C}_{p_{2}}=\delta_{p_{2}}, \\
& \mathcal{C}_{r_{2}}=\delta_{r_{2}}, \\
& \mathcal{C}_{q_{2}}=\delta_{q_{2}} .
\end{aligned}
$$

The first two terms of $\mathcal{C}_{q_{1}}$ are readily observed in chart 3 (see (3.69) and (3.70)). The other terms of $\mathcal{C}_{q_{1}}$ can only be seen in deeper charts. We skip the detail of the derivation.

The intersection matrix among $\mathcal{C}$ 's is the same one with the $p_{1}$-first case and is given by (3.41) with the same order $I, J=q_{1}, p_{0}, p_{2}, p_{1}, r_{2}, q_{2}$. Then (3.71) yields the intersection matrix of $\delta$ 's as

$$
-\delta_{I} \cdot \delta_{J}=\left(\begin{array}{cccccc}
\frac{3}{2} & 0 & 0 & 0 & 0 & -1 \\
0 & 2 & -1 & 0 & 0 & 0 \\
0 & -1 & 2 & -1 & 0 & 0 \\
0 & 0 & -1 & 2 & -1 & -1 \\
0 & 0 & 0 & -1 & 2 & 0 \\
-1 & 0 & 0 & -1 & 0 & 2
\end{array}\right)
$$




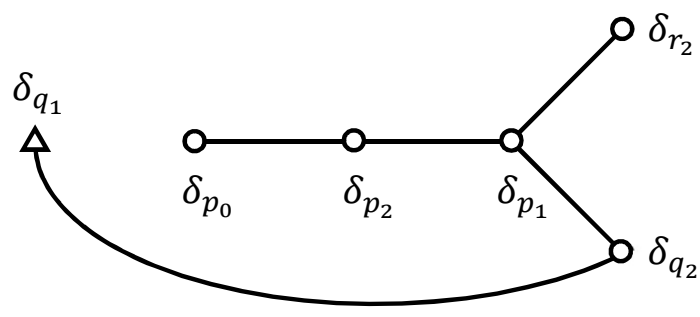

Figure 5. Incomplete intersection diagram of $\mathrm{SO}(12) \rightarrow E_{7}: q_{1}$-first $\left(p_{0} \rightarrow q_{1}\right)$ case.

This is almost the $E_{6}$ Cartan matrix except that the self-intersection number of $\delta_{q_{1}}$ is $-\frac{3}{2}$, which is expressed as a triangular node in figure 5. Thus the intersection diagram for this case is an $E_{6}$ non-Dynkin diagram.

We can search for the elements of the form $\sum_{I=q_{1}, p_{0}, p_{2}, p_{1}, r_{2}, q_{2}} n_{I} \delta_{I}$ whose square is $-\frac{3}{2}$ to find, again, that there are $16+16$ such elements, the former of which have $n_{I} \geq 0$ for all $I$, and the latter of which have $n_{I} \leq 0$ for all $I$. Thus, in this case as well, there is only one irreducible representation $(=\mathbf{3 2})$ at the singularity, showing that it is a half-hypermultiplet.

\subsection{Complete resolutions: blowing up $q_{1}$ first}

The process of the blow-ups is almost the same as that in the incomplete resolutions. A difference arises in chart $2_{z z}$, where a conifold singularity is developed at $\left(x_{2}, y_{2}, z, s\right)=$ $(0,0,0,0)$, which we denote by $q_{3}$ (shown in red in table 4 ), where the relation to the coordinates in chart $1_{z}$ is $\left(\tilde{x}_{1}, y_{1}, z, s\right)=\left(x_{2} z, y_{2} z, z, s\right)$. This is an isolated codimensiontwo singularity developed only at $s=0$. Since this is in chart $2_{z z}$, this singularity is located at $(0: 0: 1)$ on $\mathbb{P}^{2}$ emerged by the blow up at $s=0$. Therefore, it is not visible in chart $2_{z x}$ or $3_{z x *}$. Moreover, after the coordinate shift similar to (3.66), $\Psi_{z x}$ becomes identical to the incomplete case. Thus the process is the same as the incomplete case afterwards. Therefore, the only extra exceptional curve is the one arising from the small resolution of the isolated conifold singularity on $\delta_{q_{1}}$. This adds an extra node to the diagram in the figure 5 , as we show in figure 6 . We denote this new curve as $\delta_{\text {complete }}$ here. This is $E_{7}$, and the extra node again extends from $\delta_{q_{1}}$ that was the "weight" node represented by the triangle in the incomplete case. How $\delta_{\text {complete }}$ modifies the $\lim _{s \rightarrow 0} \mathcal{C}$ can be seen in the same way as explained in section 3.2.2. The result is

$$
\begin{aligned}
& \mathcal{C}_{p_{0}}=\delta_{p_{0}}, \\
& \mathcal{C}_{q_{1}}=2 \delta_{q_{1}}+2 \delta_{p_{1}}+\delta_{p_{2}}+2 \delta_{q_{2}}+\delta_{r_{2}}+\delta_{\text {complete }}, \\
& \mathcal{C}_{p_{1}}=\delta_{p_{1}}, \\
& \mathcal{C}_{p_{2}}=\delta_{p_{2}}, \\
& \mathcal{C}_{r_{2}}=\delta_{r_{2}}, \\
& \mathcal{C}_{q_{2}}=\delta_{q_{2}} .
\end{aligned}
$$

This reproduces the minus of the $D_{6}$ Cartan matrix as the intersection matrix of $\mathcal{C}$ 's if the intersections among $\delta$ 's are given by the minus of the proper $E_{7}$ Cartan matrix. Therefore, 


\begin{tabular}{|c|c|c|c|c|}
\hline & 1st blow up & 2nd blow up & 3rd blow up & 4th blow up \\
\hline (p0) $\rightarrow$ & $\begin{array}{l}\text { q1 }(-2 s: 0: 1) \rightarrow \\
p_{1}(s: 0: 1)\end{array}$ & $\begin{array}{l}\text { (43) }(0: 0: 1 ; s=0)(\operatorname{codim} .2) \rightarrow \\
p_{1}(1: 0: 0)\left(\tilde{x}_{1}=3 s\right) \rightarrow\end{array}$ & $\begin{array}{l}\text { regular } \\
P_{2}(1: 0: 0) \rightarrow \\
q_{2}(0: 0: 1) \rightarrow \\
r_{2}(1: 0:-1) \rightarrow\end{array}$ & $\begin{array}{l}\text { regular } \\
\text { regular } \\
\text { regular }\end{array}$ \\
\hline
\end{tabular}

Table 4. $\mathrm{SO}(12) \rightarrow E_{7}$ : complete case when $q_{1}$ is blown up first $\left(p_{0} \rightarrow q_{1}\right)$.

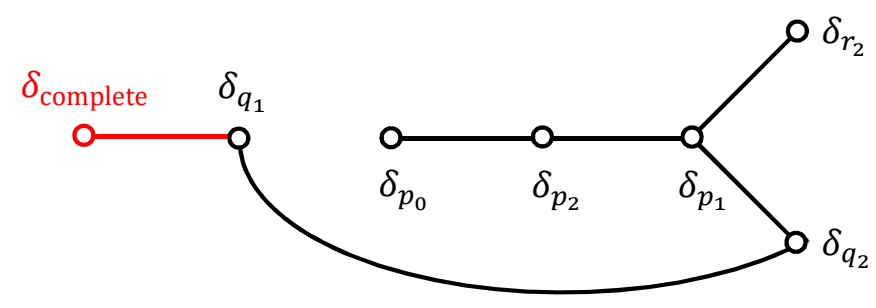

Figure 6. Complete $E_{7}$ intersection diagram of $\mathrm{SO}(12) \rightarrow E_{7}: q_{1}$-first $\left(p_{0} \rightarrow q_{1}\right)$ case.

in the complete case, $\delta_{q_{1}}$ becomes an ordinary node with self-intersection -2 as shown in figure 6 .

\subsection{Other inequivalent orderings}

So far we have considered incomplete and complete resolutions where the order of blowing up singularities is $p_{0} \rightarrow p_{1} \rightarrow p_{2}$ and $p_{0} \rightarrow q_{1}$. In the former case, we have also two codimension-one singularities $q_{1}$ and $r_{2}$, besides $p_{2}$ and $q_{2}$, after the 2 nd blow up, as shown in table 1 . Since $q_{2}$ never coincides with the other three for any value of $s$, it can be blown up independently at any stage. On the other hand, $p_{2}, q_{1}$ and $r_{2}$ become the same point $(1: 0: 0)$ on the $\mathbb{P}^{2}$ at $s=0$, so a different intersection diagram arises if we blow up $q_{1}$ or $r_{2}$ instead of $p_{2}$ after blowing up $p_{1}$. The concrete process is similar to the previous cases so we only describe the results.

$\boldsymbol{p}_{\mathbf{0}} \rightarrow \boldsymbol{p}_{\mathbf{1}} \rightarrow \boldsymbol{q}_{\mathbf{1}}$ case. If $q_{1}$ is blown up after $p_{1}$, the relations among $\mathcal{C}$ 's and $\delta$ 's are given by (the modifications via $\delta_{\text {complete }}$ in the complete case are shown in the parentheses)

$$
\begin{aligned}
& \mathcal{C}_{p_{0}}=\delta_{p_{0}}, \\
& \mathcal{C}_{p_{1}}=\delta_{p_{1}}+\delta_{q_{1}}\left(+\delta_{\text {complete }}\right), \\
& \mathcal{C}_{q_{1}}=2 \delta_{q_{1}}+\delta_{p_{2}}+\delta_{r_{2}}\left(+\delta_{\text {complete }}\right), \\
& \mathcal{C}_{p_{2}}=\delta_{p_{2}} \\
& \mathcal{C}_{r_{2}}=\delta_{r_{2}} \\
& \mathcal{C}_{q_{2}}=\delta_{q_{2}}
\end{aligned}
$$

The intersection matrix of $\mathcal{C}$ 's is the same one as before. The intersection diagrams of $\delta$ 's for incomplete / complete cases are shown in figure 7. The diagram for the incomplete case is an $E_{6}$ non-Dynkin diagram, which is similar to the $p_{0} \rightarrow q_{1}$ case. But this time there 

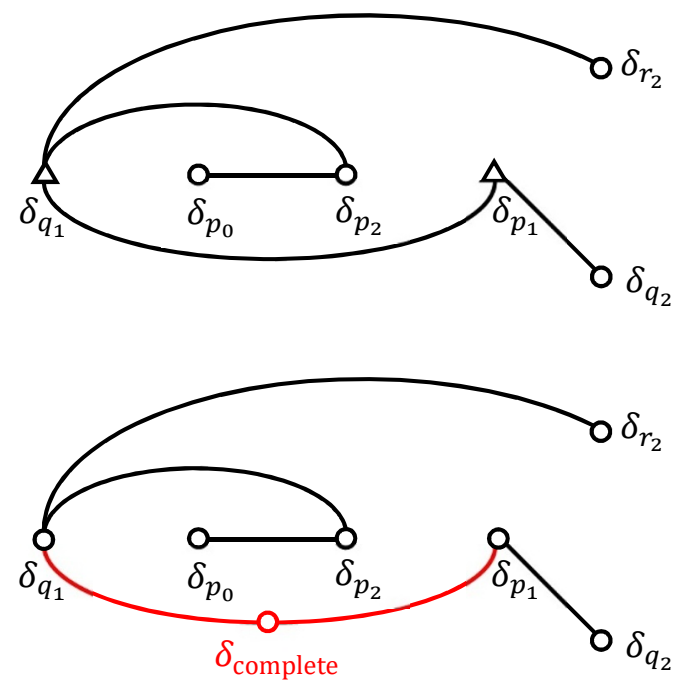

Figure 7. Incomplete/complete intersection diagrams of $\mathrm{SO}(12) \rightarrow E_{7}: p_{1}$-first $\left(p_{0} \rightarrow p_{1} \rightarrow q_{1}\right)$ case.

are two $-\frac{3}{2}$ nodes. The intersection matrix is given by

$$
-\delta_{I} \cdot \delta_{J}=\left(\begin{array}{cccccc}
\frac{3}{2} & 0 & -1 & -\frac{1}{2} & -1 & 0 \\
0 & 2 & -1 & 0 & 0 & 0 \\
-1 & -1 & 2 & 0 & 0 & 0 \\
-\frac{1}{2} & 0 & 0 & \frac{3}{2} & 0 & -1 \\
-1 & 0 & 0 & 0 & 2 & 0 \\
0 & 0 & 0 & -1 & 0 & 2
\end{array}\right)
$$

with the same order $I, J=q_{1}, p_{0}, p_{2}, p_{1}, r_{2}, q_{2}$ as before. Note that the intersection of the two $-\frac{3}{2}$ nodes is $\frac{1}{2}$.

Again, there is only one $\mathbf{3 2}$ at $s=0$ as two-cycles $J(3.44)$ satisfying $J \cdot J=-\frac{3}{2}$. For the complete case, $\delta_{\text {complete }}$ bridges the two $-\frac{3}{2}$ nodes and forms the ordinary $E_{7}$ intersection diagram.

$\boldsymbol{p}_{\mathbf{0}} \rightarrow \boldsymbol{p}_{\mathbf{1}} \rightarrow \boldsymbol{r}_{\mathbf{2}}$ case. If $r_{2}$ is blown up after $p_{1}$, the relations among $\mathcal{C}$ 's and $\delta$ 's are found to be

$$
\begin{aligned}
& \mathcal{C}_{p_{0}}=\delta_{p_{0}}+\delta_{r_{2}}\left(+\delta_{\text {complete }}\right), \\
& \mathcal{C}_{p_{1}}=\delta_{p_{1}}, \\
& \mathcal{C}_{r_{2}}=2 \delta_{r_{2}}+\delta_{p_{2}}+\delta_{q_{1}}\left(+\delta_{\text {complete }}\right), \\
& \mathcal{C}_{q_{1}}=\delta_{q_{1}}, \\
& \mathcal{C}_{p_{2}}=\delta_{p_{2}} \\
& \mathcal{C}_{q_{2}}=\delta_{q_{2}}
\end{aligned}
$$

and the intersection diagrams of $\delta$ 's are as shown in figure 8 . This time, the diagram for the incomplete case is a $D_{6}$ non-Dynkin diagram. There are two nodes with self intersections 

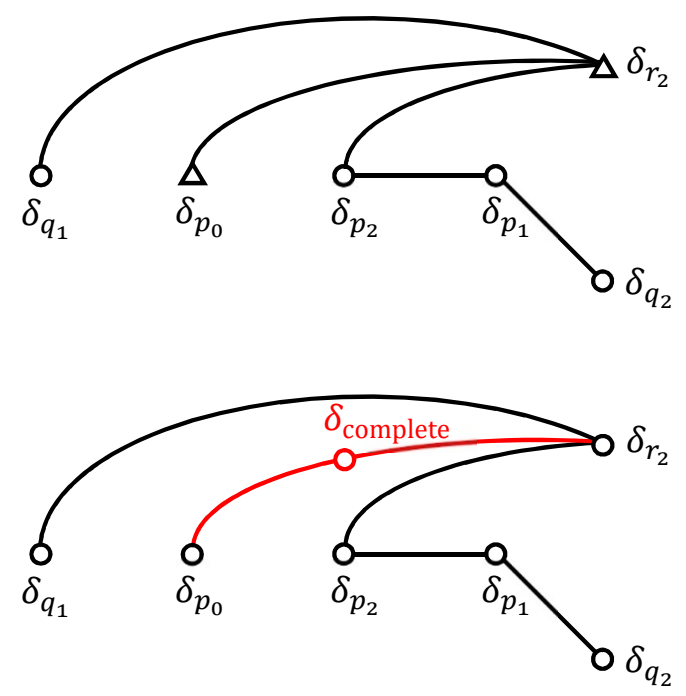

Figure 8. Incomplete/complete intersection diagrams of $\mathrm{SO}(12) \rightarrow E_{7}: p_{1}$-first $\left(p_{0} \rightarrow p_{1} \rightarrow r_{2}\right)$ case.

$-\frac{3}{2}$ and their mutual intersection is $\frac{1}{2}$. As before, only one 32 appears at $s=0$ as $J \cdot J=-\frac{3}{2}$ states. For the complete case, $\delta_{\text {complete }}$ bridges the two $-\frac{3}{2}$ nodes and forms the ordinary $E_{7}$ intersection diagram.

This exhausts all the possibilities of changing the order of the singularities we blow up.

\subsection{Comparison with the results of the M-theory Coulomb branch analysis}

In the previous sections we have obtained four distinct incomplete intersection diagrams of the fibers at the codimension-two singularity. Let us compare our results with those obtained by the M-theory Coulomb branch analysis [14].

In general, F-theory compactifications on Calabi-Yau four-folds are dual to M-theory compactifications on Calabi-Yau four-folds, which present three-dimensional $\mathcal{N}=2$ supersymmetric gauge theories. The geometry of the Calabi-Yau four-fold determines the structure of the gauge theory. In particular, the codimension-one singularity decides the gauge group, and the network of the resolution corresponds to the structure of the classical Coulomb phase since the resolution corresponds to the symmetry breaking.

We consider three-dimensional gauge theory with a gauge group $G$ and with $N_{f}$ chiral multiplets in a representation $\mathbf{R}_{f}$. We set the masses of the chiral multiplets to zero. In addition, we assume that there is no classical Chern-Simons term. The vector multiplet in the adjoint representation includes a real scalar field $\phi$. In general, the gauge group $G$ breaks to $\mathrm{U}(1)^{r}$ by the VEVs of the scalar, where $r=\operatorname{rank}(G)$. We choose the fundamental Weyl chamber as

$$
\vec{\alpha}_{i} \cdot \vec{\phi}>0
$$

where $\vec{\alpha}_{i}(i=1,2, \ldots, r)$ are the simple roots of $G . \vec{\phi}=\left(\phi^{1}, \phi^{2}, \ldots, \phi^{r}\right)$ are the VEVs in the Cartan subalgebra of $G$. 
Now we have the chiral multiplets, which make a substructure in the Coulomb branch. The Lagrangian includes the mass terms of the chiral multiplets $Q^{(f)}$ :

$$
\mathcal{L}_{\text {mass }}=\sum_{f}\left|\phi Q^{(f)}\right|^{2}=\sum_{f}\left|\vec{\phi} \cdot \vec{\omega}_{f}\right|^{2}\left|Q^{(f)}\right|^{2},
$$

where $\vec{\omega}_{f}$ are weights of $\mathbf{R}_{f}$ representation. Note that when $\vec{\phi} \cdot \vec{\omega}_{f}=0$, the corresponding matter becomes massless.

We can classify the regions of the Coulomb branch. A region is bounded by the zero loci of $\vec{\phi} \cdot \vec{\omega}_{f}$, namely, it is characterized by $\vec{\phi} \cdot \vec{\omega}_{f}>0$ or $\vec{\phi} \cdot \vec{\omega}_{f}<0$. However, not all regions are allowed since we are working on the fundamental Weyl chamber (3.77). The allowed regions of the Coulomb branch are completely classified by the decorated box graphs defined by a collection of boxes with signs (or colors) [14].

Although the analysis of [14] is based on the Coulomb branch of the three-dimensional M-theory, which basically applies to a resolution of a Calabi-Yau four-fold such as [15-20], it is interesting to compare our results with the corresponding box graphs obtained in [14] since the scalars of the five-dimensional M-theory, which are supposed to describe the resolution of a Calabi-Yau three-fold, partly comprise the three-dimensional scalars.

In [14], the intersection diagrams for the singularity enhancement $\mathrm{SO}(12) \rightarrow E_{7}$ are given in figure 33 of that paper. We can see that the intersection diagrams for incomplete resolutions shown in figures 5,7 and 8 of the present paper are the ones at the bottom, the third from the bottom and the second from the top of the left column of figure 33 of [14]. Also, the (lower) diagram in figure 2 of this paper is the diagram in the right column of figure 33 of [14]. Thus, all the four intersection diagrams we found here have corresponding box graphs obtained in [14] describing different phases of the three-dimensional gauge theory. Note that any two of them are not adjacent to each other in the seven graphs of figure 33 of [14]. This is consistent, as the two adjacent graphs of [14] are related by a flop, and the change of the order of the singularities is not a flop. Indeed, we do not have any conifold singularities until we consider a complete resolution. This is one of the differences between the resolutions in six and four dimensions.

\section{$4 \quad E_{7} \rightarrow E_{8}$}

\subsection{Incomplete resolution: blowing up $p_{2}$ first}

\subsubsection{Blowing up process}

In this case we take $f_{n+8}=s$ with setting $g_{n+12}=1, f_{8}=g_{12}=0$ in (2.17) and (2.18):

$$
\begin{aligned}
& f(z, s)=s z^{3}, \\
& g(z, s)=z^{5} .
\end{aligned}
$$

At $s \neq 0$, the orders of $f, g$ and $\Delta$ in $z$ are $(3,5,9)$, while at $s=0$, they satisfy $(\geq 4,5,10)$. Hence it describes the enhancement $I I I^{*} \rightarrow I I^{*}\left(E_{7} \rightarrow E_{8}\right)$ of the Kodaira type.

$\Phi(x, y, z, s)=0$ has a codimension-one singularity at $p_{0}=(0,0,0, s)$. The concrete process of the incomplete resolution goes as follows (an exceptional curve arising from blowing up a singularity $p$ will be denoted by $\mathcal{C}_{p}$ ): 


\section{1st blow up.}

Chart $1_{x}$

$$
\begin{aligned}
\Phi\left(x, x y_{1}, x z_{1}, s\right) & =x^{2} \Phi_{x}\left(x, y_{1}, z_{1}, s\right), \\
\Phi_{x}\left(x, y_{1}, z_{1}, s\right) & =s x^{2} z_{1}^{3}+x^{3} z_{1}^{5}+x-y_{1}^{2} . \\
\mathcal{C}_{p_{0}} \text { in } 1_{x} & : x=0, y_{1}=0 . \\
\text { Singularities } & : \text { None }
\end{aligned}
$$

Chart $1_{y} \quad \mathcal{C}_{p_{0}}$ is not visible in this chart.

Chart $1_{z}$

$$
\begin{aligned}
\Phi\left(x_{1} z, y_{1} z, z, s\right) & =z^{2} \Phi_{z}\left(x_{1}, y_{1}, z, s\right), \\
\Phi_{z}\left(x_{1}, y_{1}, z, s\right) & =z\left(x_{1} z s+x_{1}^{3}+z^{2}\right)-y_{1}^{2} . \\
\mathcal{C}_{p_{0}} \text { in } 1_{z} & : z=0, \quad y_{1}=0 .
\end{aligned}
$$

Singularities : $\left(x_{1}, y_{1}, z, s\right)=(0,0,0, s)$.

We refer to this singularity as $p_{1}$.

\section{2nd blow up.}

Chart $2_{z x}$

$$
\begin{aligned}
\Phi_{z}\left(x_{1}, x_{1} y_{2}, x_{1} z_{2}, s\right) & =x_{1}^{2} \Phi_{z x}\left(x_{1}, y_{2}, z_{2}, s\right) \\
\Phi_{z x}\left(x_{1}, y_{2}, z_{2}, s\right) & =z_{2} x_{1}\left(z_{2}\left(s+z_{2}\right)+x_{1}\right)-y_{2}^{2} . \\
\mathcal{C}_{p_{1}} \text { in } 2_{z x} & : x_{1}=0, y_{2}=0 . \\
\text { Singularities } & :\left(x_{1}, y_{2}, z_{2}, s\right)=(0,0,-s, s)\left(=q_{2}\right),(0,0,0, s)\left(=p_{2}\right) .
\end{aligned}
$$

Here we see two singularities on $\mathcal{C}_{\sqrt{ }}$ which coincide with each other at $s=0$.

Chart $2_{z y} \quad \mathcal{C}_{p_{1}}$ is not visible in this chart.

Chart $2_{z z}$

$$
\begin{aligned}
\Phi_{z}\left(x_{2} z, y_{2} z, z, s\right) & =z^{2} \Phi_{z z}\left(x_{2}, y_{2}, z, s\right) \\
\Phi_{z z}\left(x_{2}, y_{2}, z, s\right) & =z\left(s x_{2}+z x_{2}^{3}+1\right)-y_{2}^{2} . \\
\mathcal{C}_{p_{1}} \text { in } 2_{z z} & : z=0, \quad y_{2}=0 . \\
\text { Singularities } & :\left(x_{2}, y_{2}, z, s\right)=\left(-\frac{1}{s}, 0,0, s\right) .
\end{aligned}
$$

This singularity is $q_{2}$, which is also seen in chart $2_{z x}$. At this stage, we have two singularities $p_{2}$ and $q_{2}$. In this section we blow up $p_{2}$ first. We can see this singularity in chart $2_{z x}$ only, so we consider $\Phi_{z x}\left(x_{1}, y_{2}, z_{2}, s\right)$ in the next blow up. 


\section{3rd blow up.}

Chart $3_{z x x}$

$$
\begin{aligned}
\Phi_{z x}\left(x_{1}, x_{1} y_{3}, x_{1} z_{3}, s\right) & =x_{1}^{2} \Phi_{z x x}\left(x_{1}, y_{3}, z_{3}, s\right) \\
\Phi_{z x x}\left(x_{1}, y_{3}, z_{3}, s\right) & =z_{3} x_{1}\left(s z_{3}+z_{3}^{2} x_{1}+1\right)-y_{3}^{2} . \\
\mathcal{C}_{\sqrt{ } \in} \text { in } 3_{z x x} & : x_{1}=0, y_{3}=0 . \\
\text { Singularities } & :\left(x_{1}, y_{3}, z_{3}, s\right)=\left(0,0,-\frac{1}{s}, s\right)\left(=r_{3}\right),(0,0,0, s)\left(=p_{3}\right) .
\end{aligned}
$$

We name the first singularity $r_{3}$, and the second singularity $p_{3}$.

\section{Chart $3_{z x y}$ Regular.}

Chart $3_{z x z}$

$$
\begin{aligned}
\Phi_{z x}\left(x_{3} z_{2}, y_{3} z_{2}, z_{2}, s\right) & =z_{2}^{2} \Phi_{z x z}\left(x_{3}, y_{3}, z_{2}, s\right), \\
\Phi_{z x z}\left(x_{3}, y_{3}, z_{2}, s\right) & =z_{2} x_{3}\left(s+z_{2}+x_{3}\right)-y_{3}^{2} . \\
\mathcal{C}_{p_{2}} \text { in } 3_{z x z}: & z_{2}=0, y_{3}=0 . \\
\text { Singularities }: & \left(x_{3}, y_{3}, z_{2}, s\right)=(0,0,-s, s)\left(=q_{2}\right),(0,0,0, s)\left(=q_{3}\right), \\
& (-s, 0,0, s)\left(=r_{3}\right) .
\end{aligned}
$$

The first singularity is not on $\mathcal{C}_{p_{2}}$ unless $s=0$; this is $q_{2}$. We name the second singularity $q_{3}$. The third one is $r_{3}$ already seen in chart $3_{z x x}$.

When $s \rightarrow 0$, the three singularities $q_{2}, q_{3}$ and $r_{3}$ in chart $3_{z x z}$ coincide with each other, and which one we blow up next affects the proceeding process. In this section, we consider the case $q_{3}$ is blown up next. On the other hand, $p_{3}$ in chart $3_{z x x}$ is separated from these points even when $s \rightarrow 0$ and can be blown up independently. We leave the blow-up of $p_{3}$ until later and work on the blow-up of $q_{3}$.

4th blow up at $\boldsymbol{q}_{\mathbf{3}}$. We next blow up $q_{3}$. Using $\Phi_{z x z}\left(x_{3}, y_{3}, z_{2}, s\right)$, we find

Chart $4_{z x z x}$

$$
\begin{aligned}
\Phi_{z x z}\left(x_{3}, x_{3} y_{4}, x_{3} z_{4}, s\right) & =x_{3}^{2} \Phi_{z x z x}\left(x_{3}, y_{4}, z_{4}, s\right), \\
\Phi_{z x z x}\left(x_{3}, y_{4}, z_{4}, s\right) & =z_{4}\left(s+z_{4} x_{3}+x_{3}\right)-y_{4}^{2} . \\
\mathcal{C}_{q_{3}} \text { in } 4_{z x z x} & : x_{3}=0, y_{4}^{2}=s z_{4} . \\
\text { Singularities } & :\left(x_{3}, y_{4}, z_{4}, s\right)=(-s, 0,0, s) .
\end{aligned}
$$

This is $r_{3}$, which is not on $\mathcal{C}_{q_{3}}$ unless $s=0$.

Chart $4_{z x z y}$ Regular. 
Chart $4_{z x z z}$

$$
\begin{aligned}
\Phi_{z x z}\left(x_{4} z_{2}, y_{4} z_{2}, z_{2}, s\right) & =z_{2}^{2} \Phi_{z x z z}\left(x_{4}, y_{4}, z_{2}, s\right), \\
\Phi_{z x z z}\left(x_{4}, y_{4}, z_{2}, s\right)= & s x_{4}+z_{2} x_{4}\left(x_{4}+1\right)-y_{4}^{2} . \\
\mathcal{C}_{q_{3}} \text { in } 4_{z x z z} & : z_{2}=0, y_{4}^{2}=s x_{4} . \\
\text { Singularities } & :\left(x_{4}, y_{4}, z_{2}, s\right)=(0,0,-s, s) .
\end{aligned}
$$

This is $q_{2}$, which is not on $\mathcal{C}_{q_{3}}$ unless $s=0$, either. $r_{3}$ and $q_{2}$ coincide with each other at $s=0$ before blowing up $q_{3}$; but after the blow up, they are never the same point even when $s=0$. Thus we can blow them up independently.

5th blow up at $\boldsymbol{r}_{\mathbf{3}}$. To blow up $r_{3}$ in chart $4_{z x z x}$ (4.8), we shift the $x_{3}$ coordinate so that this singularity is represented as $(0,0,0, s)$ in the new coordinate $\tilde{x}_{3}$ :

$$
\Psi_{z x z x}\left(\tilde{x}_{3}, y_{4}, z_{4}, s\right) \equiv \Phi_{z x z x}\left(\tilde{x}_{3}-s, y_{4}, z_{4}, s\right) .
$$

Then it can be verified that no singularity arises in charts $5_{z x z x *}$ below. The exceptional curves are:

Chart $5_{z x z x x}$

$$
\begin{aligned}
\Psi_{z x z x}\left(\tilde{x}_{3}, \tilde{x}_{3} y_{5}, \tilde{x}_{3} z_{5}, s\right) & =\tilde{x}_{3}^{2} \Psi_{z x z x x}\left(\tilde{x}_{3}, y_{5}, z_{5}, s\right), \\
\Psi_{z x z x x}\left(\tilde{x}_{3}, y_{5}, z_{5}, s\right) & =z_{5}^{2}\left(\tilde{x}_{3}-s\right)+z_{5}-y_{5}^{2} . \\
\mathcal{C}_{r_{3}} \text { in } 5_{z x z x x} & : \tilde{x}_{3}=0, \quad y_{5}^{2}=-s z_{5}^{2}+z_{5} .
\end{aligned}
$$

Chart $5_{z x z x y}$ Invisible.

Chart $5_{z x z x z}$

$$
\begin{aligned}
\Psi_{z x z x}\left(x_{5} z_{4}, y_{5} z_{4}, z_{4}, s\right) & =z_{4}^{2} \Psi_{z x z x z}\left(x_{5}, y_{5}, z_{4}, s\right), \\
\Psi_{z x z x z}\left(x_{5}, y_{5}, z_{4}, s\right) & =-s+z_{4} x_{5}+x_{5}-y_{5}^{2} . \\
\mathcal{C}_{r_{3}} \text { in } 5_{z x z x z} & : z_{4}=0, y_{5}^{2}=x_{5}-s .
\end{aligned}
$$

5th blow up at $\boldsymbol{q}_{\mathbf{2}}$. Having resolved the singularity $r_{3}$, we turn to the resolution of $q_{2}$ in chart $4_{z x z z}(4.9)$. For this we need a different coordinate shift:

$$
\Psi_{z x z z}\left(x_{4}, y_{4}, \tilde{z}_{2}, s\right) \equiv \Phi_{z x z z}\left(x_{4}, y_{4}, \tilde{z}_{2}-s, s\right) .
$$

Then $\Psi_{z x z z}$ has a singularity at $\left(x_{4}, y_{4}, \tilde{z}_{2}, s\right)=(0,0,0, s)$. Again, charts $5_{z x z z *}$ below have no singularity. The exceptional curves are:

Chart $5_{z x z z x}$

$$
\begin{aligned}
\Psi_{z x z z}\left(x_{4}, x_{4} y_{5}, x_{4} z_{5}, s\right) & =x_{4}^{2} \Psi_{z x z z x}\left(x_{4}, y_{5}, z_{5}, s\right) \\
\Psi_{z x z z x}\left(x_{4}, y_{5}, z_{5}, s\right) & =-s+z_{5} x_{4}+z_{5}-y_{5}^{2} . \\
\mathcal{C}_{q_{2}} \text { in } 5_{z x z z x} & : x_{4}=0, \quad y_{5}^{2}=z_{5}-s
\end{aligned}
$$




\begin{tabular}{|c|c|c|c|c|c|}
\hline & 1st blow up & 2nd blow up & 3rd blow up & 4th blow up & 5th blow up \\
\hline$p_{0} \rightarrow$ & $p_{1}(0: 0: 1) \rightarrow$ & $\begin{array}{l}p_{2}(1: 0: 0) \rightarrow \\
q_{2}(1: 0:-s)\end{array}$ & $\begin{array}{l}p_{3}(1: 0: 0) \rightarrow \\
q_{3}(0: 0: 1) \rightarrow \\
q_{2}(0: 0: 1)\left(z_{2}=-s\right) \\
r_{3}(-s: 0: 1)\end{array}$ & $\begin{array}{l}\text { regular } \\
\text { regular } \\
q_{2}(0: 0: 1)\left(z_{2}=-s\right) \rightarrow \\
r_{3}(1: 0: 0)\left(x_{3}=-s\right) \rightarrow\end{array}$ & $\begin{array}{l}\text { regular } \\
\text { regular }\end{array}$ \\
\hline
\end{tabular}

Table 5. $E_{7} \rightarrow E_{8}$ : incomplete case when $p_{2}$ is blown up first $\left(p_{0} \rightarrow p_{1} \rightarrow p_{2} \rightarrow q_{3}\right)$.

Chart $5_{z x z z y} \quad$ We omit the details.

Chart $5_{z x z z z}$

$$
\begin{aligned}
\Psi_{z x z z}\left(x_{5} \tilde{z}_{2}, y_{5} \tilde{z}_{2}, \tilde{z}_{2}, s\right) & =\tilde{z}_{2}^{2} \Psi_{z x z z z}\left(x_{5}, y_{5}, \tilde{z}_{2}, s\right) \\
\Psi_{z x z z z}\left(x_{5}, y_{5}, \tilde{z}_{2}, s\right) & =x_{5}^{2}\left(-s+\tilde{z}_{2}\right)+x_{5}-y_{5}^{2} \\
\mathcal{C}_{q_{2}} \text { in } 5_{z x z z z} & : \tilde{z}_{2}=0, \quad y_{5}^{2}=-s x_{5}^{2}+x_{5}
\end{aligned}
$$

4th blow up at $\boldsymbol{p}_{\mathbf{3}}$. Let us return to chart $3_{z x x}$ and blow up the remaining $p_{3}(4.6)$.

Chart $4_{z x x x}$

$$
\begin{aligned}
\Phi_{z x x}\left(x_{1}, x_{1} y_{4}, x_{1} z_{4}, s\right) & =x_{1}^{2} \Phi_{z x x x}\left(x_{1}, y_{4}, z_{4}, s\right) \\
\Phi_{z x x x}\left(x_{1}, y_{4}, z_{4}, s\right) & =s z_{4}^{2} x_{1}+z_{4}^{3} x_{1}^{3}+z_{4}-y_{4}^{2} \\
\mathcal{C}_{p_{3}} \text { in } 4_{z x x x} & : x_{1}=0, \quad y_{4}^{2}=z_{4} .
\end{aligned}
$$

Singularities : None.

Chart $4_{z x x y}$ Regular.

Chart $4_{z x x z}$

$$
\begin{aligned}
\Phi_{z x x}\left(x_{4} z_{3}, y_{4} z_{3}, z_{3}, s\right) & =z_{3}^{2} \Phi_{z x x z}\left(x_{4}, y_{4}, z_{3}, s\right) \\
\Phi_{z x x z}\left(x_{4}, y_{4}, z_{3}, s\right) & =s z_{3} x_{4}+z_{3}^{3} x_{4}^{2}+x_{4}-y_{4}^{2} . \\
\mathcal{C}_{p_{3}} \text { in } 4_{z x x z} & : z_{3}=0, y_{4}^{2}=x_{4} . \\
\text { Singularities } & :\left(x_{4}, y_{4}, z_{3}, s\right)=\left(0,0,-\frac{1}{s}, s\right) .
\end{aligned}
$$

This singularity is not on $\mathcal{C}_{p_{3}}$ even when $s=0$; this is $r_{3}$ (see (4.6)) and is blown up as in (4.11) and (4.12).

The whole process of the incomplete resolution of the codimension-two singularity enhancement from $E_{7}$ to $E_{8}$ is summarized in table 5.

\subsubsection{Intersection diagram at $s=0$}

Exceptional curves $\delta_{I}$ at $s=0$ are defined from $\mathcal{C}_{I}\left(I \in\left\{p_{0}, p_{1}, p_{2}, q_{3}, r_{3}, q_{2}, p_{3}\right\}\right)$. One can see from the explicit blowing up process that the intersection patterns of $\mathcal{C}$ 's and $\delta$ 's are 
$E_{7}$ and $A_{7}$, respectively (see figure 9 below). As explained in section 3.1.3, the $s \rightarrow 0$ limit of $\mathcal{C}_{I}$ is derived through the careful lift-ups of $\mathcal{C}_{I}$ and $\delta_{I}$ from the chart where they are originally defined to the charts arise via the subsequent blow-ups. In this case, we have

$$
\begin{aligned}
& \mathcal{C}_{p_{0}}=\delta_{p_{0}}, \\
& \mathcal{C}_{p_{1}}=\delta_{p_{1}}, \\
& \mathcal{C}_{p_{2}}=\delta_{p_{2}}, \\
& \mathcal{C}_{q_{3}}=2 \delta_{q_{3}}+\delta_{r_{3}}+\delta_{q_{2}}, \\
& \mathcal{C}_{r_{3}}=\delta_{r_{3}}, \\
& \mathcal{C}_{q_{2}}=\delta_{q_{2}}, \\
& \mathcal{C}_{p_{3}}=\delta_{p_{3}} .
\end{aligned}
$$

The intersection matrix of $\mathcal{C}$ 's is the minus of the ordinary $E_{7}$ Cartan matrix

$$
-\mathcal{C}_{I} \cdot \mathcal{C}_{J}=\left(\begin{array}{ccccccc}
2 & -1 & 0 & 0 & 0 & 0 & 0 \\
-1 & 2 & -1 & 0 & 0 & 0 & 0 \\
0 & -1 & 2 & -1 & 0 & 0 & -1 \\
0 & 0 & -1 & 2 & -1 & 0 & 0 \\
0 & 0 & 0 & -1 & 2 & -1 & 0 \\
0 & 0 & 0 & 0 & -1 & 2 & 0 \\
0 & 0 & -1 & 0 & 0 & 0 & 2
\end{array}\right)
$$

where $I, J=p_{0}, p_{3}, p_{2}, q_{3}, p_{1}, q_{2}, r_{3}$ in this order. Then the relations (4.18) imply that the intersection matrix of $\delta$ 's is

$$
-\delta_{I} \cdot \delta_{J}=\left(\begin{array}{ccccccc}
2 & -1 & 0 & 0 & 0 & 0 & 0 \\
-1 & 2 & -1 & 0 & 0 & 0 & 0 \\
0 & -1 & 2 & 0 & 0 & 0 & -1 \\
0 & 0 & 0 & \frac{3}{2} & 0 & -1 & -1 \\
0 & 0 & 0 & 0 & 2 & -1 & 0 \\
0 & 0 & 0 & -1 & -1 & 2 & 0 \\
0 & 0 & -1 & -1 & 0 & 0 & 2
\end{array}\right)
$$

This is the minus of the $A_{7}$ Cartan matrix except that one of the $\delta$ 's $\left(=\delta_{q_{3}}\right)$ has selfintersection $-\frac{3}{2}$, which equals to the minus of the length squared of a weight in the $\mathbf{5 6}$ representation of $E_{7}$. The result is shown in figure 9 . For the two-cycles at $s=0$

$$
J \equiv \sum_{I=p_{0}, p_{3}, p_{2}, q_{3}, p_{1}, q_{2}, r_{3}} n_{I} \delta_{I} \quad\left(n_{I} \in \mathbb{Z}\right),
$$

one can show by using (4.20) that

$$
\begin{aligned}
& \sharp(J \cdot J=-2)=126, \\
& \sharp\left(J \cdot J=-\frac{3}{2}\right)=56 .
\end{aligned}
$$

They respectively are the adjoint (without Cartan part) and $\mathbf{5 6}$ representations of $E_{7}$. The latter consists of 28 elements with $n_{I} \geq 0$ for all $I$ and 28 elements with $n_{I} \leq 0$ for all $I$. Again, there is only a single $\mathbf{5 6}$ representation, indicating that it is a half-hypermultiplet. 

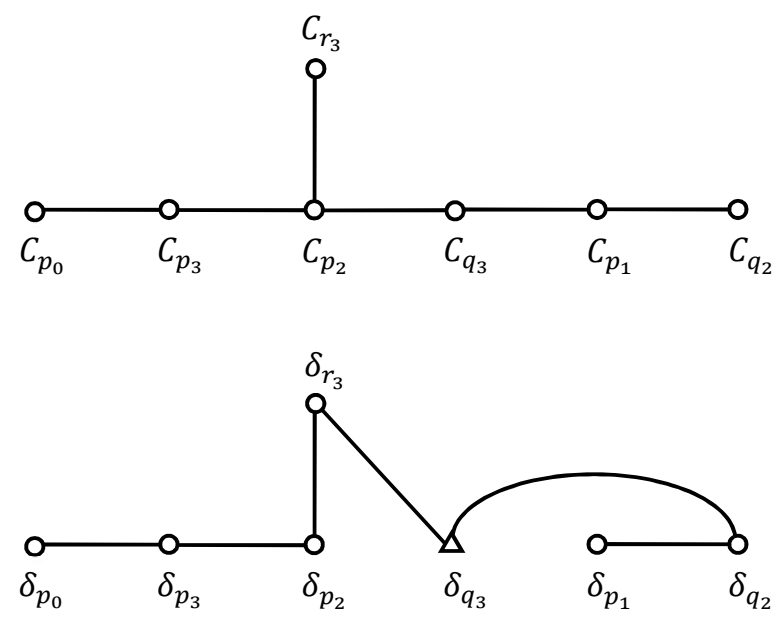

Figure 9. Generic $E_{7}$ intersection diagram at $s \neq 0$ (upper) and incomplete intersection diagram at $s=0$ (lower) of $E_{7} \rightarrow E_{8}: p_{2}$-first $\left(p_{0} \rightarrow p_{1} \rightarrow p_{2} \rightarrow q_{3}\right)$ case.

\begin{tabular}{|c|c|c|c|}
\hline & 1st blow up & 2nd blow up & 3rd blow up \\
\hline \multirow[t]{3}{*}{ p0 $\rightarrow$} & $p_{1}(0: 0: 1) \rightarrow$ & $\begin{array}{l}p_{2}(1: 0: 0) \rightarrow \\
q_{2}\left(1: 0:-s^{2}\right)\end{array}$ & $\begin{array}{l}p_{3}(1: 0: 0) \rightarrow \\
q_{3}(0: 0: 1) \rightarrow \\
q_{2}(0: 0: 1)\left(z_{2}=-s^{2}\right) \\
r_{3}\left(-s^{2}: 0: 1\right)\end{array}$ \\
\hline & \multicolumn{2}{|l|}{ 4th blow up } & 5 th blow up \\
\hline & \multicolumn{2}{|c|}{$\begin{array}{l}\text { regular } \\
\text { ra }(1: 0:-1 ; s=0)(\operatorname{codim} .2) \rightarrow \\
\left(r_{2}\right)(0: 0: 1)\left(z_{2}=-s^{2}\right) \rightarrow \\
r_{3}(1: 0: 0)\left(x_{3}=-s^{2}\right) \rightarrow\end{array}$} & $\begin{array}{l}\text { regular } \\
\text { regular } \\
\text { regular }\end{array}$ \\
\hline
\end{tabular}

Table 6. $E_{7} \rightarrow E_{8}$ : complete case when $p_{2}$ is blown up first $\left(p_{0} \rightarrow p_{1} \rightarrow p_{2} \rightarrow q_{3}\right)$.

\subsection{Complete resolution}

We will now consider the complete resolution. This can be achieved by taking $f_{n+8}=s^{2}$ instead of $s$. This amounts to replacing $s$ in (4.1) with $s^{2}$. Similarly to the previous sections, we find an additional isolated codimension-two conifold singularity after we blow up $q_{3}$. As shown in red in table 6 , this new singularity, which we denote by $r_{4}$, arises at $(1: 0:-1)$ on the $\mathbb{P}^{2}$ particularly at $s=0$. This adds an extra node to the incomplete intersection diagram to form the correct $E_{8}$ Dynkin diagram as we show in figure 10 . To see how the intersection matrix is modified, we repeat the argument given in section 3.2.2. By carefully lifting up $\mathcal{C}$ 's and $\delta$ 's into the local coordinate system of the small resolution, 


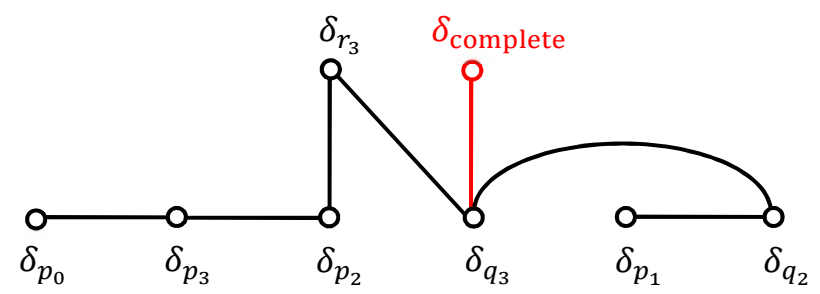

Figure 10. Complete $E_{8}$ intersection diagram of $E_{7} \rightarrow E_{8}: p_{2}$-first $\left(p_{0} \rightarrow p_{1} \rightarrow p_{2} \rightarrow q_{3}\right)$ case.

we find the modified relations

$$
\begin{aligned}
& \mathcal{C}_{p_{0}}=\delta_{p_{0}} \\
& \mathcal{C}_{p_{1}}=\delta_{p_{1}} \\
& \mathcal{C}_{p_{2}}=\delta_{p_{2}} \\
& \mathcal{C}_{q_{3}}=2 \delta_{q_{3}}+\delta_{r_{3}}+\delta_{q_{2}}+\delta_{\text {complete }}, \\
& \mathcal{C}_{r_{3}}=\delta_{r_{3}} \\
& \mathcal{C}_{q_{2}}=\delta_{q_{2}} \\
& \mathcal{C}_{p_{3}}=\delta_{p_{3}} .
\end{aligned}
$$

One can then verify that: if the intersection matrix of these eight $\delta$ 's is the minus of the ordinary $E_{8}$ Cartan matrix, the intersection matrix of $\mathcal{C}$ 's (4.19) is reproduced. Therefore, the node $\delta_{q_{3}}$, which was formerly represented by a triangle in figure 9 , is now an ordinary node consisting of the root system of $E_{8}$ as in figure 10 .

\subsection{Incomplete/complete resolutions: blowing up $q_{2}$ first}

As we did for $\mathrm{SO}(12) \rightarrow E_{7}$, we can change the order of the blow-ups to obtain a different intersection diagram. For instance, we can choose $q_{2}$ instead of $p_{2}$ for the 3rd blow up. The procedures are analogous to the previous cases so we report only the results.

For the incomplete resolution, the whole process of the blow-ups is as shown in table 7 . We use the same notation for $\mathcal{C}$ 's (and hence for $\delta$ 's) as was used in the $p_{2}$-first case. Then the intersection pattern of $\mathcal{C}$ 's for the $q_{2}$-first case is the same $E_{7}$ Dynkin diagram with the $p_{2}$-first case given in figure 9 and their intersection matrix is also the same with (4.19). The intersection pattern of $\delta$ 's, however, is different from the one $\left(A_{7}\right)$ given in figure 9 . This time, it is $E_{7}$ (see figure 11 below).

One can verify the relations

$$
\begin{aligned}
& \mathcal{C}_{p_{0}}=\delta_{p_{0}}+\delta_{q_{2}}, \\
& \mathcal{C}_{p_{1}}=\delta_{p_{1}}, \\
& \mathcal{C}_{q_{2}}=2 \delta_{q_{2}}+2 \delta_{p_{2}}+2 \delta_{p_{3}}+\delta_{q_{3}}+\delta_{r_{3}}, \\
& \mathcal{C}_{p_{2}}=\delta_{p_{2}}, \\
& \mathcal{C}_{p_{3}}=\delta_{p_{3}}, \\
& \mathcal{C}_{q_{3}}=\delta_{q_{3}}, \\
& \mathcal{C}_{r_{3}}=\delta_{r_{3}} .
\end{aligned}
$$




\begin{tabular}{|c|c|c|c|c|c|}
\hline & 1st blow up & 2nd blow up & 3rd blow up & 4th blow up & 5 th blow up \\
\hline$p_{0} \rightarrow$ & $p_{1}(0: 0: 1) \rightarrow$ & $\begin{array}{l}q_{2}(1: 0:-s) \rightarrow \\
p_{2}(1: 0: 0)\end{array}$ & $\begin{array}{l}\text { regular } \\
p_{2}(0: 0: 1)\left(\tilde{z}_{2}=s\right) \rightarrow\end{array}$ & $\begin{array}{l}p_{3}(1: 0: 0) \rightarrow \\
q_{3}(0: 0: 1) \rightarrow \\
r_{3}(1: 0:-1) \rightarrow\end{array}$ & $\begin{array}{l}\text { regular } \\
\text { regular } \\
\text { regular }\end{array}$ \\
\hline
\end{tabular}

Table 7. $E_{7} \rightarrow E_{8}$ : incomplete case when $q_{2}$ is blown up first $\left(p_{0} \rightarrow p_{1} \rightarrow q_{2}\right)$.

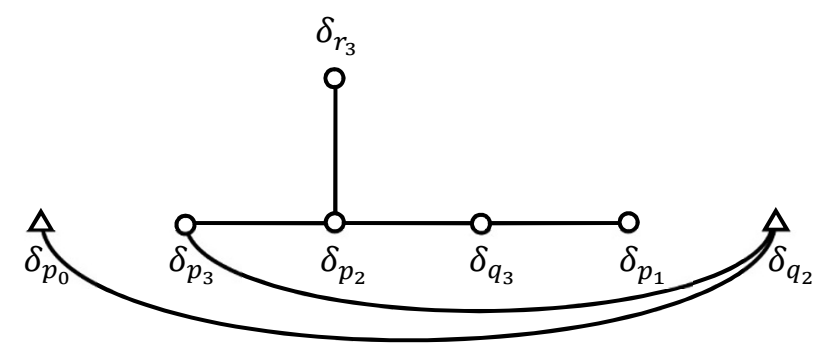

Figure 11. Incomplete intersection diagram of $E_{7} \rightarrow E_{8}: q_{2}$-first $\left(p_{0} \rightarrow p_{1} \rightarrow q_{2}\right)$ case.

Then the intersection matrix among $\delta$ 's has two $-\frac{3}{2}$ nodes as shown in figure 11 . The intersection among these two nodes is $\frac{1}{2}$. As is the same as the previous examples, one can form $28+28$ different linear combinations of $\delta$ 's with non-negative and non-positive integer coefficients such that they have self-intersection $-\frac{3}{2}$, giving a single $\mathbf{5 6}$ representation.

In the complete case, the 3rd blow up at $q_{2}$ does not end with a smooth configuration but an isolated codimension-two conifold singularity remains at the intersection of $\delta_{p_{0}}$ and $\delta_{q_{2}}$ at $s=0$ (see table 8 ). By a small resolution of this, the relations are modified as

$$
\begin{aligned}
& \mathcal{C}_{p_{0}}=\delta_{p_{0}}+\delta_{q_{2}}+\delta_{\text {complete }}, \\
& \mathcal{C}_{p_{1}}=\delta_{p_{1}}, \\
& \mathcal{C}_{q_{2}}=2 \delta_{q_{2}}+2 \delta_{p_{2}}+2 \delta_{p_{3}}+\delta_{q_{3}}+\delta_{r_{3}}+\delta_{\text {complete }}, \\
& \mathcal{C}_{p_{2}}=\delta_{p_{2}}, \\
& \mathcal{C}_{p_{3}}=\delta_{p_{3}}, \\
& \mathcal{C}_{q_{3}}=\delta_{q_{3}}, \\
& \mathcal{C}_{r_{3}}=\delta_{r_{3}} .
\end{aligned}
$$

Demanding that the intersection matrix among $\delta$ 's is the minus of the proper $E_{8}$ Cartan matrix is consistent with the intersection matrix among $\mathcal{C}$ 's. As a result, we obtain figure 12.

\subsection{Other inequivalent orderings}

Let us summarize what other types of intersection diagrams are obtained for the enhancement $E_{7} \rightarrow E_{8}$ if we choose other orderings of the blow-ups. So far, we have derived the intersection diagrams for blow-ups with orders $p_{0} \rightarrow p_{1} \rightarrow p_{2} \rightarrow q_{3}$ and $p_{0} \rightarrow p_{1} \rightarrow q_{2}$. As we can see in the column "3rd blow up" in table 5 , after blowing up $p_{2}$, the three singular points $q_{3}, q_{2}$ and $r_{3}$ become an identical point $(0: 0: 1)$ on the $\mathbb{P}^{2}$ at $s=0$ 


\begin{tabular}{|c|c|c|c|}
\hline & 1st blow up & 2nd blow up & 3rd blow up \\
\hline \multirow[t]{3}{*}{ (p) $\rightarrow$} & $p_{1}(0: 0: 1) \rightarrow$ & $\begin{array}{l}q_{2}(1: 0:-s) \rightarrow \\
p_{2}(1: 0: 0)\end{array}$ & $\begin{array}{l}p_{4}(1: 0: 0 ; s=0)(\operatorname{codim} .2) \rightarrow \\
p_{2}(0: 0: 1)\left(\tilde{z}_{2}=s\right) \rightarrow\end{array}$ \\
\hline & 4th blow up & 5 th blow up & \\
\hline & $\begin{array}{l}\text { regular } \\
\begin{array}{l}p_{3}(1: 0: 0) \rightarrow \\
(93(0: 0: 1) \rightarrow \\
r_{3}(1: 0:-1) \rightarrow\end{array}\end{array}$ & $\begin{array}{l}\text { regular } \\
\text { regular } \\
\text { regular }\end{array}$ & \\
\hline
\end{tabular}

Table 8. $E_{7} \rightarrow E_{8}:$ complete case when $q_{2}$ is blown up first $\left(p_{0} \rightarrow p_{1} \rightarrow q_{2}\right)$.

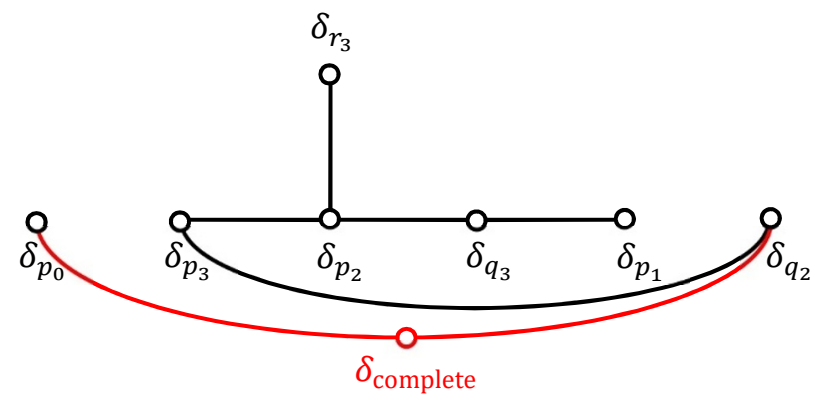

Figure 12. Complete $E_{8}$ intersection diagram of $E_{7} \rightarrow E_{8}: q_{2}$-first $\left(p_{0} \rightarrow p_{1} \rightarrow q_{2}\right)$ case.

(see also (4.7)). Therefore, besides the case when $q_{3}$ is blown up after $p_{2}$ as discussed in sections 4.1 and $4.2,{ }^{6}$ there are two other options: we can blow up either $q_{2}$ or $r_{3}$ after the blow up of $p_{2}$.

$\boldsymbol{p}_{\mathbf{0}} \rightarrow \boldsymbol{p}_{\mathbf{1}} \rightarrow \boldsymbol{p}_{\mathbf{2}} \rightarrow \boldsymbol{q}_{\mathbf{2}}$ case. If we blow up $q_{2}$ after $p_{2}$, the relations between $\mathcal{C}$ 's and $\delta$ 's are given by

$$
\begin{aligned}
& \mathcal{C}_{p_{0}}=\delta_{p_{0}}, \\
& \mathcal{C}_{p_{1}}=\delta_{p_{1}}, \\
& \mathcal{C}_{p_{2}}=\delta_{p_{2}}+\delta_{q_{2}}\left(+\delta_{\text {complete }}\right), \\
& \mathcal{C}_{q_{2}}=2 \delta_{q_{2}}+\delta_{q_{3}}+\delta_{r_{3}}\left(+\delta_{\text {complete }}\right), \\
& \mathcal{C}_{q_{3}}=\delta_{q_{3}}, \\
& \mathcal{C}_{r_{3}}=\delta_{r_{3}}, \\
& \mathcal{C}_{p_{3}}=\delta_{p_{3}} .
\end{aligned}
$$

The intersection diagram of $\mathcal{C}$ 's is the $E_{7}$ Dynkin diagram as before. The intersection diagrams of $\delta$ 's for incomplete / complete cases are shown in figure 13. For the incomplete case, it is an $E_{7}$ non-Dynkin diagram with two $-\frac{3}{2}$ nodes (the intersection among them is

\footnotetext{
${ }^{6} p_{3}$ is always a different point from the three and hence can be blown up independently at any stage.
} 

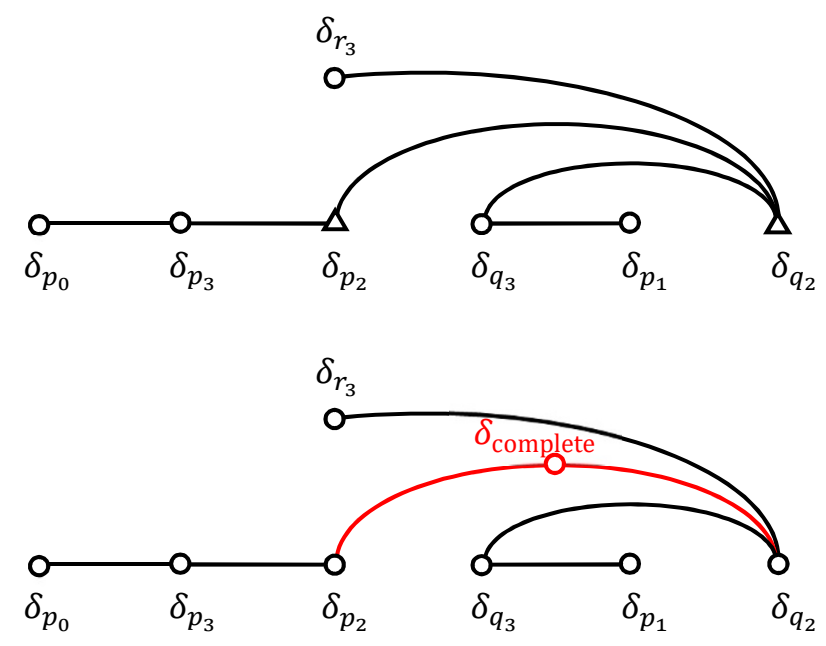

Figure 13. Incomplete/complete intersection diagrams of $E_{7} \rightarrow E_{8}: p_{2}$-first $\left(p_{0} \rightarrow p_{1} \rightarrow p_{2} \rightarrow q_{2}\right)$ case.

$\frac{1}{2}$ ), while for the complete case, it is proper $E_{8}$ Dynkin diagram. Again, there is only one $\mathbf{5 6}$ at $s=0$ as two-cycles $J(3.44)$ with $J \cdot J=-\frac{3}{2}$.

$\boldsymbol{p}_{\mathbf{0}} \rightarrow \boldsymbol{p}_{\mathbf{1}} \rightarrow \boldsymbol{p}_{\mathbf{2}} \rightarrow \boldsymbol{r}_{\mathbf{3}}$ case. If we blow up $r_{3}$ after $p_{2}$, the relations between $\mathcal{C}$ 's and $\delta$ 's are given by

$$
\begin{aligned}
& \mathcal{C}_{p_{0}}=\delta_{p_{0}}, \\
& \mathcal{C}_{p_{1}}=\delta_{p_{1}}+\delta_{r_{3}}\left(+\delta_{\text {complete }}\right), \\
& \mathcal{C}_{p_{2}}=\delta_{p_{2}}, \\
& \mathcal{C}_{r_{3}}=2 \delta_{r_{3}}+\delta_{q_{2}}+\delta_{q_{3}}\left(+\delta_{\text {complete }}\right), \\
& \mathcal{C}_{q_{2}}=\delta_{q_{2}}, \\
& \mathcal{C}_{q_{3}}=\delta_{q_{3}}, \\
& \mathcal{C}_{p_{3}}=\delta_{p_{3}} .
\end{aligned}
$$

The intersection diagrams are shown in figure 14. The diagram for the incomplete case is a $D_{7}$ non-Dynkin diagram with two $-\frac{3}{2}$ nodes (their intersection is $\frac{1}{2}$ ). Again, there is only one 56 at $s=0$.

This exhausts all the possibilities of changing the order of the singularities we blow up. We obtained four sets of incomplete/complete intersection diagrams. Again, each of them corresponds to a box graph on every other row of figure 44 in [14]. For the $p_{2}$-first cases, figure 9 (with figure 10) is equivalent to the one in the right column: figure 13 and figure 14 respectively are the fourth and sixth ones from the bottom in the left column. For the $q_{2}$-first case, figure 11 (with figure 12) is the second one from the bottom in the left column. 

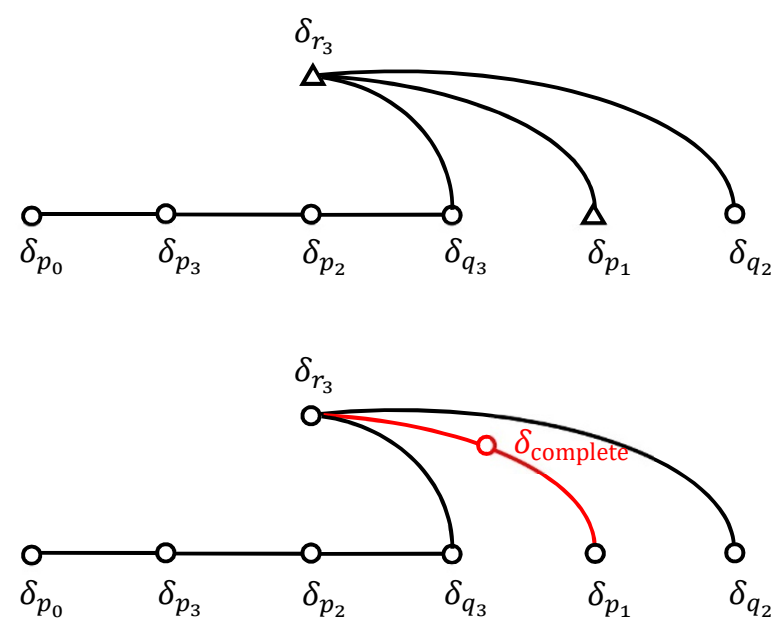

Figure 14. Incomplete/complete intersection diagrams of $E_{7} \rightarrow E_{8}: p_{2}$-first $\left(p_{0} \rightarrow p_{1} \rightarrow p_{2} \rightarrow r_{3}\right)$ case.

\section{Conclusions}

We have investigated the resolutions of codimension-two enhanced singularities from $\mathrm{SO}(12)$ to $E_{7}$ and from $E_{7}$ to $E_{8}$ in six-dimensional F-theory, where a half-hypermultiplet locally arises for generic complex structures achieving them. A half-hypermultiplet only occurs associated with a Lie algebra allowing a pseudo-real representation, and the above are the two of the three cases in the list of six-dimensional F-theory compactifications [7] that exhibit half-hypermultiplets in the massless matter spectrum. As was already observed in the enhancement from $\mathrm{SU}(6)$ to $E_{6}$ in [11], we have confirmed that the resolution process does not generically yield as many number of exceptional curves as naively expected from the Kodaira classification of the codimension-one singularities. In the present paper, we have observed similar features such as non-Dynkin intersection diagrams and half-integral intersection numbers of exceptional fibers. Then we have found that the exceptional fibers at the enhanced point form extremal rays of the cone of the positive weights of the relevant pseudo-real representation, explaining why a half-hyper multiplet arises there.

We have also found that a variety of different intersection diagrams of exceptional curves are obtained by altering the ordering of the singularities blown up in the process. They correspond to different "phases" of the three-dimensional M-theory. We have obtained, for both $\mathrm{SO}(12) \rightarrow E_{7}$ and $E_{7} \rightarrow E_{8}$, the intersection diagram on every other row of the figures in [14], but not all of them. The phases corresponding to the diagrams we obtained are not the ones related by a flop.

We have presented detailed derivations of the intersection diagrams of the exceptional fibers at the singularity enhanced points. In particular, we have described how an exceptional curve is lifted up on the chart arising due to the subsequent blowing-up process. By carefully examining whether an exceptional curve contains another arising afterwards as a part, we have obtained the intersection matrices as above.

In the complete resolutions, where the colliding brane approaches the stack of branes as $\mathrm{O}\left(s^{2}\right)$, we have obtained the full Dynkin diagrams of the group $G$ as the intersection 
diagram of the fibers at the enhanced point. The extra codimension-two singularity is always a conifold singularity, as was found in the previous example [11].

Although we have studied in this paper the explicit resolutions of the singularities in six dimensions, the technologies we have developed here can also be used in more general settings such as codimension-three singularities in Calabi-Yau four-folds, with or without sections. (In the latter, one may consider the Jacobian fibrations. See e.g. [29].)

It would also be interesting to perform a similar analysis for a singularity with higher rank enhancement. In particular, it has been expected [10] that a codimension-three singularity enhancement from $\mathrm{SU}(5)$ to $E_{7}$ could yield, without monodromies and with appropriate $G$-fluxes, the three-generation spectrum of the $E_{7} /\left(\mathrm{SU}(5) \times \mathrm{U}(1)^{3}\right)$ supersymmetric coset model [30]. Such a codimension-two singularity enhancement in six dimensions was already studied in [31]. The codimension-three case with a $\mathbb{Z}_{2}$ or a larger monodromy was considered in e.g. [32], but was concluded to be not very useful for their purposes. The box graph analysis of [14], on the other hand, predicts the existence of the phases without monodromies, which will serve as basis for models of family unification in F-theory. We hope to come back to this issue elsewhere.

Finally, massless matter generation in F-theory may also be explained by string junctions stretched between various $(p, q)$ 7-branes near the intersections. Very recently, a new pictorial method to keep track of non-localness of F-theory 7-branes has been developed by drawing a "dessin" on the base of the elliptic fibration [33, 34], which may help understanding how the difference of the resolutions is affected by the geometry near the enhanced point.

\section{Acknowledgments}

We thank H. Hayashi, Y. Kimura, H. Otsuka and S. Schafer-Nameki for valuable discussions.

\section{A Symplectic Majorana-Weyl spinors, pseudo-real representations and half-hypermultiplets}

\section{A.1 Symplectic Majorana-Weyl spinors}

In $\operatorname{six}(=5+1)$ dimensions, consider the Dirac equation

$$
\left(i \gamma^{\mu} \partial_{\mu}-m\right) \psi=0
$$

The complex conjugate equation

$$
\left(-i \gamma^{\mu *} \partial_{\mu}-m\right) \psi^{*}=0
$$

can be written in terms of the charge conjugation $\psi^{c}$ defined by

$$
\psi^{c} \equiv B \psi^{*}
$$


with

$$
B \gamma^{\mu *} B^{-1}=-\gamma^{\mu}
$$

as

$$
\left(i \gamma^{\mu} \partial_{\mu}-m\right) \psi^{c}=0
$$

This is the same Dirac equation as that $\psi$ obeys. If one could impose the constraint $\psi^{c}=\psi$ on $\psi$, one could define a Majorana spinor, but in six dimensions one can not as, if one could do so,

$$
\psi=\psi^{c}=B \psi^{*}=B\left(B \psi^{*}\right)^{*}=B B^{*} \psi,
$$

but

$$
B B^{*}=-1
$$

in six dimensions. Alternatively, one can impose on two Weyl spinors $\psi_{1}, \psi_{2}$ the constraint

$$
\psi_{1}^{c}=+\psi_{2}, \quad \psi_{2}^{c}=-\psi_{1} .
$$

They are called symplectic Majorana-Weyl spinors. Note that in $\operatorname{six}(=5+1)$-dimensions the charge conjugation operation does not flip the chirality so that one can define symplectic Majorana- Weyl spinors.

\section{A.2 Pseudo-real representation and symplectic Majorana condition}

Let us examine whether one can consistently impose this constraint on a representation space of a Lie group. Let $G$ be a compact Lie group and $\rho$ be its representation on a complex $2 n$-dimensional vector space $V$ :

$$
\rho: G \rightarrow G L_{\mathbb{C}}(V) \text { homomorphism. }
$$

We say $\rho$ is a pseudo-real representation if there exists $P \in G L_{\mathbb{C}}(V)$ such that

$$
P(\rho(g))^{*} P^{-1}=\rho(g)
$$

for any $g \in G .{ }^{*}$ denotes the complex conjugation.

If

$$
v^{\prime}=\rho(g) v
$$

then

$$
\begin{aligned}
P v^{*} & =\left(P(\rho(g))^{*} P^{-1}\right) P v^{*} \\
& =\rho(g) P v^{*} .
\end{aligned}
$$

Therefore $P v^{*}$ also transforms as $\mathbf{2 n}$. 
If $\rho(g)$ can be embedded in $\operatorname{Sp}(2 n)$, we have

$$
\begin{aligned}
\rho(g)^{T} \Omega_{2 n} \rho(g) & =\Omega_{2 n}, \\
\Omega_{2 n} & =\left(\begin{array}{cc}
0 & -1_{n} \\
1_{n} & 0
\end{array}\right) .
\end{aligned}
$$

Moreover, if $\rho$ is a unitary representation: $\rho(g)^{-1}=\rho(g)^{\dagger}$, we find

$$
\rho(g)=\Omega_{2 n} \rho(g)^{*} \Omega_{2 n}^{-1} .
$$

Thus we can take

$$
P=\Omega_{2 n}
$$

Let us construct such a vector $v$ by using symplectic Majorana(-Weyl) spinors. Let $\psi_{1}$, $\psi_{2}$ be a pair of $n$-component column vectors consisting of $n$ symplectic Majorana(-Weyl) spinors, and $v$ be a stack of them. Then

$$
\begin{aligned}
v & =\left(\begin{array}{l}
\psi_{1} \\
\psi_{2}
\end{array}\right) \\
& =\left(\begin{array}{c}
-\psi_{2}^{c} \\
\psi_{1}^{c}
\end{array}\right) \\
& =\left(\begin{array}{cc}
0 & -1_{n} \\
1_{n} & 0
\end{array}\right)\left(\begin{array}{l}
\psi_{1}^{c} \\
\psi_{2}^{c}
\end{array}\right) \\
& =\Omega_{2 n}\left(\begin{array}{c}
B \psi_{1}^{*} \\
B \psi_{2}^{*}
\end{array}\right) \\
& =\Omega_{2 n}\left(B \cdot 1_{2 n}\right) v^{*} .
\end{aligned}
$$

In general, this is not consistent as $v$ transforms as a $\mathbf{2 n}$ representation whereas $v^{*}$ as a $\overline{\mathbf{2 n}}$ representation. However, for a pseudo-real representation (A.14), we obtain

$$
\begin{aligned}
\rho(g) v & =\Omega_{2 n} \rho(g)^{*} \Omega_{2 n}^{-1} \cdot \Omega_{2 n}\left(B \cdot 1_{2 n}\right) v^{*} \\
& =\Omega_{2 n}\left(B \cdot 1_{2 n}\right) \rho(g)^{*} v^{*},
\end{aligned}
$$

which agrees with (A.16). Therefore, for a pseudo-real representation a $2 n$-dimensional representation space can be constructed from $n$ pairs of symplectic Majorana-Weyl spinors.

\section{A.3 $\frac{2 \mathrm{n}}{2}$ hypermultiplets vs. $2 \mathrm{n} \frac{1}{2}$ hypermultiplets}

In the previous section we have seen that among $2 n$ Weyl fermions in hypermultiplets transforming as a pseudo-real $\mathbf{2 n}$ representation one half $(=n)$ of them can be expressed as the complex conjugates of the other half $(=n)$. In this section we will show that this can be viewed as a restriction of the degrees of freedom of $2 n$ (Weyl fermions of) hypermultiplets to $2 n$ ("half-Weyl" fermions of) half-hypermultiplets. 
We take

$$
\begin{aligned}
& \Gamma^{0}=\sigma_{3} \otimes \sigma_{3} \otimes i \sigma_{2}, \\
& \Gamma^{1}=\sigma_{3} \otimes \sigma_{3} \otimes \sigma_{1}, \\
& \Gamma^{2}=\sigma_{3} \otimes \sigma_{1} \otimes 1, \\
& \Gamma^{3}=\sigma_{3} \otimes \sigma_{2} \otimes 1, \\
& \Gamma^{4}=\sigma_{1} \otimes 1 \otimes 1, \\
& \Gamma^{5}=\sigma_{2} \otimes 1 \otimes 1
\end{aligned}
$$

as a realization of the gamma matrices. As the matrix $B$ satisfying (A.4), we can have

$$
B=\Gamma^{0} \Gamma^{1} \Gamma^{2} \Gamma^{4}
$$

Since

$$
\Gamma^{0} \Gamma^{1} \Gamma^{2}=\sigma_{3} \otimes \sigma_{1} \otimes \sigma_{3},
$$

we can write $B$ in a block form as

$$
B=\left(\begin{array}{cc}
b & 0 \\
0 & -b
\end{array}\right)\left(\begin{array}{ll}
0 & 1 \\
1 & 0
\end{array}\right),
$$

where

$$
\sigma_{1} \otimes \sigma_{3} \equiv b
$$

$\mathbf{1}$ is the $4 \times 4$ unit matrix.

Since the chirality in six dimensions is defined by

$$
\Gamma^{\sharp} \equiv-\Gamma^{0} \Gamma^{1} \Gamma^{2} \Gamma^{3} \Gamma^{4} \Gamma^{5} \equiv\left(\sigma_{3} \otimes \mathbf{1}\right)\left(\mathbf{1} \otimes \gamma_{5}\right),
$$

the eigenvalues of $\gamma_{5}$ and $\left(\sigma_{3} \otimes \mathbf{1}\right)$ are correlated with each other in a six-dimensional spinor with a definite chirality. For instance, if $\Gamma^{\sharp}=+1,\left(\gamma_{5}, \sigma_{3}\right)=(+,+)$ or $(-,-)$. Thus if we write

$$
\psi_{i}=\left(\begin{array}{c}
\phi_{i} \\
\chi_{i}
\end{array}\right) \quad(i=1,2),
$$

this is a decomposition with respect to the four-dimensional chirality. From (A.21), we have

$$
\begin{aligned}
\psi_{i}^{c} & =B \psi_{i}^{*} \\
& =\left(\begin{array}{cc}
b & 0 \\
0 & -b
\end{array}\right)\left(\begin{array}{ll}
0 & \mathbf{1} \\
\mathbf{1} & 0
\end{array}\right)\left(\begin{array}{l}
\phi_{i}^{*} \\
\chi_{i}^{*}
\end{array}\right) \\
& =\left(\begin{array}{r}
b \chi_{i}^{*} \\
-b \phi_{i}^{*}
\end{array}\right) .
\end{aligned}
$$

Therefore, if a collection of $n$ spinors $\psi_{2}$ are written as $\psi_{1}^{c}$, the relations $\psi_{2}=\psi_{1}^{c}$ and $\psi_{1}=-\psi_{2}^{c}$ imply

$$
\chi_{2}=-b \phi_{1}^{*}, \quad \chi_{1}=+b \phi_{2}^{*} .
$$

Thus the lower component of each of the $2 n$ Weyl spinors can be expressed in terms of the upper component. 


\section{A.4 Restriction on the complex scalars}

Let $v_{i}(i=1,2)$ be a pair of $n$ complex scalars and

$$
\left(\begin{array}{l}
v_{1} \\
v_{2}
\end{array}\right)
$$

be in the $\mathbf{2 n}$ pseudo-real representation of $G$. In order to similarly define $v_{i}^{c}$ such that

$$
v_{2}=v_{1}^{c}, \quad v_{1}=-v_{2}^{c}
$$

by

$$
v_{i}^{c} \equiv U v_{i}^{*}
$$

for some $U$ satisfying $U^{*} U=-1$, we recall that a hypermultiplet has two complex scalars transforming in the identical representation. Let $v_{i}^{(1)}$ and $v_{i}^{(2)}$ be such two scalars, then writing $v_{i}=\left(\begin{array}{c}v_{i}^{(1)} \\ v_{i}^{(2)}\end{array}\right)$, we define

$$
\begin{aligned}
v_{i}^{c} & =\left(\begin{array}{c}
v_{i}^{(1) c} \\
v_{i}^{(2) c}
\end{array}\right) \\
& \equiv U\left(\begin{array}{c}
v_{i}^{(1) *} \\
v_{i}^{(2) *}
\end{array}\right),
\end{aligned}
$$

where

$$
U \equiv\left(\begin{array}{cc}
0 & -1 \\
1 & 0
\end{array}\right)
$$

is a $90^{\circ} \mathrm{U}(1)_{R}$ rotation. Then $U^{*} U=-1$, and (A.28) can be imposed. With this definition of $v_{i}^{c}$, one can also reduce the degrees of freedom of the complex scalars in a hypermultiplet.

\section{B Summary of SU(6) $\rightarrow E_{6}$}

In this appendix we summarize the results of the analysis [11] of the codimension-two enhancement $\mathrm{SU}(6) \rightarrow E_{6}$. Consider

$$
\Phi=-y^{2}+x^{3}+\left(t_{r}^{2}+3 t_{r} z\right) x^{2}+\left(2 t_{r} z^{2}+3 z^{3}\right) x+z^{4} .
$$

One can verify that $\Phi$ has an $\mathrm{SU}(6)$ singularity at $(x, y, z)$ for fixed $t_{r} \neq 0$, and this is enhanced to an $E_{6}$ singularity for $t_{r}=0$. $\Phi$ (B.1) can be obtained by putting $h_{n+2-r}=\frac{1}{\sqrt{3}}$, $H_{n+4-r}=-\frac{1}{2}, u_{r+4}=\frac{1}{2}, f_{n+8-r}=f_{8}=g_{12}=0$ in (2.8) and (2.9) and making a change of variables.

If we take $t_{r}=s$, we are led to the incomplete resolution. The process is summarized in table 9. After the codimension-one blow up at the singularity $p_{0}$ of $\Phi$ (B.1), we obtain two exceptional curves $\mathcal{C}_{1 \pm}$ at fixed $s \neq 0$, which come on top of each other into a single 


\begin{tabular}{|l|l|l|l|}
\hline & 1st blow up & 2nd blow up & 3rd blow up \\
\hline$p_{0} \rightarrow$ & $P_{1}(0: 0: 1) \rightarrow$ & $P_{2}(1: 0:-s) \rightarrow$ & regular \\
\hline
\end{tabular}

Table 9. $\mathrm{SU}(6) \rightarrow E_{6}$ : incomplete case.
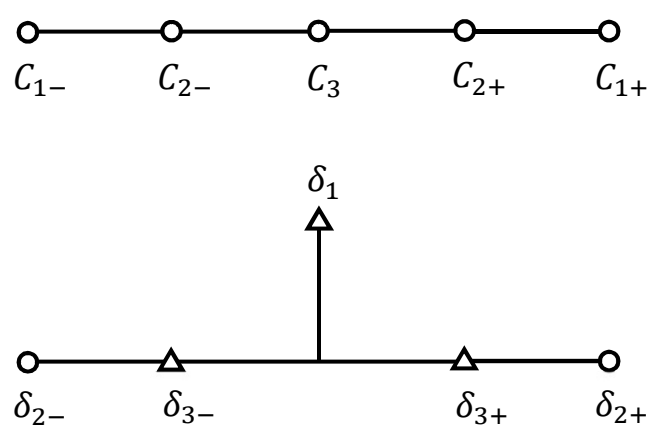

Figure 15. Generic intersection diagram at $s \neq 0$ (upper) and incomplete intersection diagram at $s=0$ (lower) of $\mathrm{SU}(6) \rightarrow E_{6}$.

curve $\delta_{1}$ at $s=0$. These two curves intersect at a singularity $p_{1}$, which forms a singular line along the $s$ direction. We perform a codimension-one blow up along this line to find, again, two exceptional curves $\mathcal{C}_{2 \pm}$ at $s \neq 0$, which becomes $\delta_{2 \pm}$ at $s=0$. The intersection of $\mathcal{C}_{2 \pm}$ is again another singularity $p_{2}$. We then blow up the singularity line $p_{2}$ to get a regular curve $\mathcal{C}_{3}$ at $s \neq 0$, which splits into two curves $\delta_{3 \pm}$ at $s=0$.

In the incomplete case, this is the end. The relations among $\mathcal{C}$ 's and $\delta$ 's are given by

$$
\begin{aligned}
& \mathcal{C}_{1 \pm}=\delta_{1}+\delta_{3 \pm}, \\
& \mathcal{C}_{2 \pm}=\delta_{2 \pm}, \\
& \mathcal{C}_{3}=\delta_{3+}+\delta_{3-} .
\end{aligned}
$$

One can derive these relations in the same way as explained in section 3.1.3. Their intersection diagrams are shown in figure 15.

For the complete resolution, we take $t_{r}=s^{2}$. In this case, as is shown in table 10, we have an additional codimension-two isolated conifold singularity after the 3rd blow up.

The relations (B.2) are modified to

$$
\begin{aligned}
& \mathcal{C}_{1 \pm}=\delta_{1}+\delta_{3 \pm}+\delta_{\text {complete }} \\
& \mathcal{C}_{2 \pm}=\delta_{2 \pm} \\
& \mathcal{C}_{3}=\delta_{3+}+\delta_{3-}+\delta_{\text {complete }}
\end{aligned}
$$

This result is obtained by following the analysis explained in section 3.2.2. The intersection diagram of these six $\delta$ 's is the proper $E_{6}$ Dynkin diagram as in figure 16. 


\begin{tabular}{|l|l|l|l|l|}
\hline & 1st blow up & 2nd blow up & 3rd blow up & 4th blow up \\
\hline$p_{0} \rightarrow$ & $p_{1}(0: 0: 1) \rightarrow$ & $p_{2}(1: 0:-w) \rightarrow$ & $p_{3}(1: 0: 0 ; s=0)($ codim.2) $\rightarrow$ & regular \\
\hline
\end{tabular}

Table 10. $\mathrm{SU}(6) \rightarrow E_{6}$ : complete case.

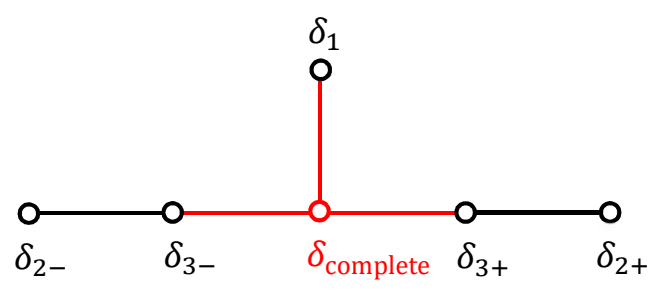

Figure 16. Complete $E_{6}$ intersection diagram of $\mathrm{SU}(6) \rightarrow E_{6}$.

\section{Small resolution of a conifold singularity}

In this appendix, we give a brief review of the small resolution (see, e.g., [28]). A conifold $\mathcal{M}$ is a three dimensional space given by the polynomial in $\mathbb{C}^{4}$

$$
X_{1} X_{4}-X_{2} X_{3}=0
$$

It has a singularity at $\left(X_{1}, X_{2}, X_{3}, X_{4}\right)=(0,0,0,0)$, which is called the conifold singularity. In general, an isolated singularity on a hypersurface in $\mathbb{C}^{4}$ can be resolved by inserting $\mathbb{P}^{3}$ in the ambient space $\mathbb{C}^{4}$. For the conifold singularity, it is equivalent to inserting $\mathbb{P}^{1} \times \mathbb{P}^{1}$ on the conifold $\mathcal{M}$. However, it is sufficient to insert only one $\mathbb{P}^{1}$ to resolve the conifold singularity. This process is called the small resolution. Inserting $\mathbb{P}^{1}$ at the origin of $\mathcal{M}$ is given by

$$
\breve{\mathcal{M}}=\left\{\left(X_{1}, X_{2}, X_{3}, X_{4}\right) \times\left(y_{1}: y_{2}\right) \mid\left[\begin{array}{ll}
X_{1} & X_{2} \\
X_{3} & X_{4}
\end{array}\right]\left[\begin{array}{l}
y_{1} \\
y_{2}
\end{array}\right]=\left[\begin{array}{l}
0 \\
0
\end{array}\right]\right\} .
$$

Let us write $M=\left[\begin{array}{ll}X_{1} & X_{2} \\ X_{3} & X_{4}\end{array}\right]$. Since $\left(y_{1}: y_{2}\right) \neq(0,0), \operatorname{det} M=0$. This gives the defining equation of the conifold (C.1). Furthermore, if

$$
\left(X_{1}, X_{2}, X_{3}, X_{4}\right)\left\{\begin{array}{l}
\neq(0,0,0,0) \Rightarrow \operatorname{rank} M=1 \Rightarrow\left(y_{1}: y_{2}\right) \text { is determined } \\
=(0,0,0,0) \Rightarrow \operatorname{rank} M=0 \Rightarrow\left(y_{1}: y_{2}\right) \text { is undetermined }
\end{array}\right.
$$

which means that $\mathbb{P}^{1}=\left(y_{1}: y_{2}\right)$ is inserted only at the origin. As a result, $\breve{\mathcal{M}}$ is regular, since $\partial_{X_{1}}\left(X_{1} y_{1}+X_{2} y_{2}\right)=y_{1}=0$ and $\partial_{X_{2}}\left(X_{1} y_{1}+X_{2} y_{2}\right)=y_{2}=0$ are not compatible.

$\breve{\mathcal{M}}$ is covered by two local coordinate patches. If $y_{1} \neq 0$, we can solve (C.2) as

$$
X_{1}=-\frac{y_{2}}{y_{1}} X_{2}, X_{3}=-\frac{y_{2}}{y_{1}} X_{4},
$$

and hence

$$
\left(X_{1}, X_{2}, X_{3}, X_{4}\right)=\left(-\lambda X_{2}, X_{2},-\lambda X_{4}, X_{4}\right) \quad \text { with } \lambda \equiv \frac{y_{2}}{y_{1}}
$$


We call this patch $H_{+}$. Local coordinates of $H_{+}$are $\left(X_{2}, X_{4}, \lambda\right)$ and the inserted $\mathbb{P}^{1}=\left(y_{1}\right.$ : $\left.y_{2}\right)$ is located at

$$
\left(X_{2}, X_{4}, \lambda\right)=(0,0, \lambda)
$$

If $y_{2} \neq 0$, we can solve (C.2) as

$$
X_{2}=-\frac{y_{1}}{y_{2}} X_{1}, X_{4}=-\frac{y_{1}}{y_{2}} X_{3}
$$

and hence

$$
\left(X_{1}, X_{2}, X_{3}, X_{4}\right)=\left(X_{1},-\mu X_{1}, X_{3},-\mu X_{3}\right) \quad \text { with } \mu \equiv \frac{y_{1}}{y_{2}} .
$$

We call this patch $H_{-}$. Local coordinates of $H_{-}$are $\left(X_{1}, X_{3}, \mu\right)$ and the inserted $\mathbb{P}^{1}$ is

$$
\left(X_{1}, X_{3}, \mu\right)=(0,0, \mu)
$$

Open Access. This article is distributed under the terms of the Creative Commons Attribution License (CC-BY 4.0), which permits any use, distribution and reproduction in any medium, provided the original author(s) and source are credited.

\section{References}

[1] C. Vafa, Evidence for F-theory, Nucl. Phys. B 469 (1996) 403 [hep-th/9602022] [InSPIRE].

[2] E. Witten, Strong coupling expansion of Calabi-Yau compactification, Nucl. Phys. B 471 (1996) 135 [hep-th/9602070] [inSPIRE].

[3] R. Blumenhagen, B. Körs, D. Lüst and T. Ott, The standard model from stable intersecting brane world orbifolds, Nucl. Phys. B 616 (2001) 3 [hep-th/0107138] [INSPIRE].

[4] R. Blumenhagen, M. Cvetič, D. Lüst, R. Richter and T. Weigand, Non-perturbative Yukawa Couplings from String Instantons, Phys. Rev. Lett. 100 (2008) 061602 [arXiv:0707.1871] [INSPIRE].

[5] D.R. Morrison and C. Vafa, Compactifications of F-theory on Calabi-Yau threefolds. 1, Nucl. Phys. B 473 (1996) 74 [hep-th/9602114] [InSPIRE].

[6] D.R. Morrison and C. Vafa, Compactifications of F-theory on Calabi-Yau threefolds. 2., Nucl. Phys. B 476 (1996) 437 [hep-th/9603161] [INSPIRE].

[7] M. Bershadsky, K.A. Intriligator, S. Kachru, D.R. Morrison, V. Sadov and C. Vafa, Geometric singularities and enhanced gauge symmetries, Nucl. Phys. B 481 (1996) 215 [hep-th/9605200] [INSPIRE].

[8] S.H. Katz and C. Vafa, Matter from geometry, Nucl. Phys. B 497 (1997) 146 [hep-th/9606086] [INSPIRE].

[9] T. Tani, Matter from string junction, Nucl. Phys. B 602 (2001) 434 [INSPIRE].

[10] S. Mizoguchi, F-theory Family Unification, JHEP 07 (2014) 018 [arXiv:1403.7066] [INSPIRE].

[11] D.R. Morrison and W. Taylor, Matter and singularities, JHEP 01 (2012) 022 [arXiv:1106.3563] [INSPIRE]. 
[12] M. Günaydin, G. Sierra and P.K. Townsend, Exceptional Supergravity Theories and the MAGIC Square, Phys. Lett. B 133 (1983) 72 [InSPIRE].

[13] M. Günaydin, G. Sierra and P.K. Townsend, The Geometry of $N=2$ Maxwell-Einstein Supergravity and Jordan Algebras, Nucl. Phys. B 242 (1984) 244 [InSPIRE].

[14] H. Hayashi, C. Lawrie, D.R. Morrison and S. Schäfer-Nameki, Box Graphs and Singular Fibers, JHEP 05 (2014) 048 [arXiv:1402.2653] [INSPIRE].

[15] M. Esole and S.-T. Yau, Small resolutions of SU(5)-models in F-theory, Adv. Theor. Math. Phys. 17 (2013) 1195 [arXiv:1107.0733] [INSPIRE].

[16] J. Marsano and S. Schäfer-Nameki, Yukawas, G-flux, and Spectral Covers from Resolved Calabi-Yau's, JHEP 11 (2011) 098 [arXiv:1108.1794] [INSPIRE].

[17] M. Esole, S.-H. Shao and S.-T. Yau, Singularities and Gauge Theory Phases, Adv. Theor. Math. Phys. 19 (2015) 1183 [arXiv:1402.6331] [INSPIRE].

[18] M. Esole, S.-H. Shao and S.-T. Yau, Singularities and Gauge Theory Phases II, Adv. Theor. Math. Phys. 20 (2016) 683 [arXiv:1407.1867] [INSPIRE].

[19] A.P. Braun and S. Schäfer-Nameki, Box Graphs and Resolutions I, Nucl. Phys. B 905 (2016) 447 [arXiv: 1407.3520] [INSPIRE].

[20] A.P. Braun and S. Schäfer-Nameki, Box Graphs and Resolutions II: From Coulomb Phases to Fiber Faces, Nucl. Phys. B 905 (2016) 480 [arXiv:1511.01801] [InSPIRE].

[21] H. Pinkham, Factorization of birational maps in dimension 3, in Proceedings of Symposia in Pure Mathematics. Vol. 40: Singularities. Part 2, P. Orlik eds., AMS Press, New York U.S.A. (1983), pg. 343.

[22] M. Reid, Minimal models of canonical 3-folds, in Advanced Studies in Pure Mathematics. Vol. 1: Algebraic Varieties and Analytic Varieties, S. Iitaka eds., Kinokuniya, Tokyo Japan (1983), pg. 131.

[23] S. Katz and D.R. Morrison, Gorenstein threefold singularities with small resolutions via invariant theory for Weyl groups, J. Alg. Geom. 1 (1992) 449 [alg-geom/9202002].

[24] R. Friedman, J. Morgan and E. Witten, Vector bundles and F-theory, Commun. Math. Phys. 187 (1997) 679 [hep-th/9701162] [INSPIRE].

[25] R. Donagi and M. Wijnholt, Model Building with F-theory, Adv. Theor. Math. Phys. 15 (2011) 1237 [arXiv: 0802.2969] [INSPIRE].

[26] S. Mizoguchi and T. Tani, Looijenga's weighted projective space, Tate's algorithm and Mordell-Weil Lattice in F-theory and heterotic string theory, JHEP 11 (2016) 053 [arXiv: 1607.07280] [INSPIRE].

[27] K. Oguiso and T. Shioda, The Mordell-Weil Lattice of a Rational Elliptic Surface, Comment. Math. Univ. St. Pauli. 40 (1991) 83.

[28] P. Candelas and X.C. de la Ossa, Comments on Conifolds, Nucl. Phys. B 342 (1990) 246 [INSPIRE].

[29] Y. Kimura, Discrete Gauge Groups in F-theory Models on Genus-One Fibered Calabi-Yau 4-folds without Section, JHEP 04 (2017) 168 [arXiv: 1608.07219] [INSPIRE].

[30] T. Kugo and T. Yanagida, Unification of Families Based on a Coset Space E7/SU(5) $\times \mathrm{SU}(3) \times \mathrm{U}(1)$, Phys. Lett. B $134(1984) 313$ [inSPIRE]. 
[31] S. Mizoguchi and T. Tani, Anomaly-free multiple singularity enhancement in F-theory, PTEP 2016 (2016) 073B05 [arXiv: 1508.07423] [INSPIRE].

[32] J.J. Heckman, A. Tavanfar and C. Vafa, The Point of $E_{8}$ in F-theory GUTs, JHEP 08 (2010) 040 [arXiv: 0906.0581] [INSPIRE].

[33] S. Fukuchi, N. Kan, S. Mizoguchi and H. Tashiro, Dessin d'enfant and a description of mutually nonlocal 7-branes without using branch cuts, Phys. Rev. D 100 (2019) 126025 [arXiv: 1808.04135] [INSPIRE].

[34] S. Fukuchi, N. Kan, R. Kuramochi, S. Mizoguchi and H. Tashiro, More on a dessin on the base: Kodaira exceptional fibers and mutually (non-)local branes, Phys. Lett. B 803 (2020) 135333 [arXiv: 1912.02974] [INSPIRE]. 